Алгебра и анализ

Tом 20 (2008), № 1
St. Petersburg Math. J. Vol. 20 (2009), No. 1, Pages 23-57 S 1061-0022(08)01036-4

Article electronically published on November 13, 2008

\title{
WEIGHT ELEMENTS OF CHEVALLEY GROUPS
}

\author{
N. A. VAVILOV
}

\begin{abstract}
The paper is devoted to a detailed study of some remarkable semisimple elements of (extended) Chevalley groups that are diagonalizable over the ground field - the weight elements. These are the conjugates of certain semisimple elements $h_{\omega}(\varepsilon)$ of extended Chevalley groups $\bar{G}=\bar{G}(\Phi, K)$, where $\omega$ is a weight of the dual root system $\Phi^{\vee}$ and $\varepsilon \in K^{*}$. In the adjoint case the $h_{\omega}(\varepsilon)^{\prime}$ 's were defined by Chevalley himself and in the simply connected case they were constructed by Berman and Moody. The conjugates of $h_{\omega}(\varepsilon)$ are called weight elements of type $\omega$. Various constructions of weight elements are discussed in the paper, in particular, their action in irreducible rational representations and weight elements induced on a regularly embedded Chevalley subgroup by the conjugation action of a larger Chevalley group. It is proved that for a given $x \in \bar{G}$ all elements $x(\varepsilon)=x h_{\omega}(\varepsilon) x^{-1}, \varepsilon \in K^{*}$, apart maybe from a finite number of them, lie in the same Bruhat coset $\bar{B} w \bar{B}$, where $w$ is an involution of the Weyl group $W=W(\Phi)$. The elements $h_{\omega}(\varepsilon)$ are particularly important when $\omega=\varpi_{i}$ is a microweight of $\Phi^{\vee}$. The main result of the paper is a calculation of the factors of the Bruhat decomposition of microweight elements $x(\varepsilon)$ for the case where $\omega=\varpi_{i}$. It turns out that all nontrivial $x(\varepsilon)$ 's lie in the same Bruhat coset $\bar{B} w \bar{B}$, where $w$ is a product of reflections in pairwise strictly orthogonal roots $\gamma_{1}, \ldots, \gamma_{r+s}$. Moreover, if among these roots $r$ are long and $s$ are short, then $r+2 s$ does not exceed the width of the unipotent radical of the $i$ th maximal parabolic subgroup in $\bar{G}$. A version of this result was first announced in a paper by the author in Soviet Mathematics: Doklady in 1988. From a technical viewpoint, this amounts to the determination of Borel orbits of a Levi factor of a parabolic subgroup with Abelian unipotent radical and generalizes some results of Richardson, Röhrle, and Steinberg. These results are instrumental in the description of overgroups of a split maximal torus and in the recent papers by the author and V. Nesterov on the geometry of tori.
\end{abstract}

\section{INTRODUCTION}

Let $\Phi$ be a reduced irreducible root system in an $l$-dimensional Euclidean space $V$. The inner product in $V$ is denoted by ( , ). For two roots $\alpha, \beta \in \Phi$, denote by $\langle\beta, \alpha\rangle=$ $2(\beta, \alpha) /(\alpha, \alpha)=\left(\beta, \alpha^{\vee}\right)$ the corresponding Cartan number, where $\alpha^{\vee}=2 \alpha /(\alpha, \alpha)$ is the dual root. Let $\Phi^{\vee}=\left\{\alpha^{\vee}, \alpha \in \Phi\right\}$ denote the dual root system. By $Q(\Phi)$ we denote the root lattice generated by all $\alpha \in \Phi$. Furthermore, $P(\Phi)$ is the weight lattice consisting of all $\omega \in V$ such that $\left(\alpha^{\vee}, \omega\right) \in \mathbb{Z}$ for all $\alpha^{\vee} \in \Phi^{\vee}$. Fix a fundamental subsystem $\Pi=\left\{\alpha_{1}, \ldots, \alpha_{l}\right\}$ in $\Phi$. Let $\Phi^{+}$and $\Phi^{-}$be the corresponding sets of positive and negative roots, respectively. The choice of $\Pi$ endows $P(\Phi)$ with a partial order as follows: $\lambda \succeq \mu$ if $\lambda-\mu=\sum m_{i} \alpha_{i}$, where all $m_{i} \geq 0$.

Next, let $K$ be a field; $P$ a lattice lying between $Q(\Phi)$ and $P(\Phi) ; G=G(\Phi, K)$ a Chevalley group of type $\Phi, P$ over $K$; and $T=T(\Phi, K)$ a split maximal torus in $G$. In the case where $P=P(\Phi)$, the group $G=G_{\mathrm{sc}}$ is said to be simply connected and if

2000 Mathematics Subject Classification. Primary 20G15.

Key words and phrases. Chevalley groups, semisimple elements, Bruhat decomposition, microweights, Borel orbits, parabolic subgroups with Abelian unipotent radical. 
$P=Q(\Phi)$, then the group $G=G_{\text {ad }}$ is adjoint. Usually we suppose that the group $G$ is simply connected. For a root $\alpha \in \Phi$ and an element $\xi \in K$ we denote by $x_{\alpha}(\xi)$ the corresponding elementary root unipotent in $G$. For a fixed $\alpha$ the set of all $x_{\alpha}$ 's forms an elementary unipotent root subgroup $X_{\alpha}=\left\{x_{\alpha}(\xi), \xi \in K\right\}$.

Let $\omega \in P\left(\Phi^{\vee}\right)$. Then, by definition, $(\alpha, \omega) \in \mathbb{Z}$ for all $\alpha \in \Phi$. Thus, for any $\varepsilon \in K^{*}$ we can define a $K$-character $\chi=\chi_{\omega, \varepsilon}$ of the root lattice $Q(\Phi)$ by $\chi_{\omega, \varepsilon}(\alpha)=\varepsilon^{(\alpha, \omega)}$. Now we can consider the diagonal automorphism associated with this character (see [34, 43]). We denote by $h_{\omega}(\varepsilon)$ an element conjugation by which realizes this diagonal automorphism. In other words, this element should commute with all elements in $T$ and satisfy the following commutator relation:

$$
h_{\omega}(\varepsilon) x_{\alpha}(\xi) h_{\omega}(\varepsilon)^{-1}=x_{\alpha}\left(\varepsilon^{(\alpha, \omega)} \xi\right)
$$

for all $\alpha \in \Phi, \xi \in K$. In general, these elements do not belong to $G$, but belong to its diagonal extension $\bar{G}$, the extended Chevalley group, which stands in the same relation to the Chevalley group itself as the general linear group $\operatorname{GL}(n, K)$ does to the special linear group $\mathrm{SL}(n, K)$, or the general symplectic group $\mathrm{GSp}(2 l, K)$ does to the symplectic group $\operatorname{Sp}(2 l, K)$. We call an element $h$ of $\bar{G}$ a weight element of type $\omega$ if it is conjugate to some $h_{\omega}(\varepsilon)$.

Our purpose in this paper is to study the behavior of these elements and their conjugates in some detail. For the adjoint groups, weight elements were studied by C. Chevalley himself in his original paper [36. For the simply connected case, the situation is much more complicated and the corresponding elements are defined by equation (0) only modulo a central factor. The first explicit uniform construction of the group $\bar{G}_{\mathrm{sc}}-$ and thus of the weight elements - was given by S. Berman and R. Moody in [40]. Of course, for most (all?) cases the groups themselves had been known before as the groups of similarities of the corresponding bilinear or multilinear forms, or as Clifford groups. The classical cases are well known; see the references in 76 for the exceptional ones. In this paper we give some further constructions of these elements. In particular, in Theorem 1 we describe the action of these elements in the irreducible representations of the group $G$. In Theorem 2 we study which weight elements are induced by conjugation by the elements of a Chevalley group $G$ on regularly embedded Chevalley subgroups, in particular on the irreducible Levi factors of parabolic subgroups of $G$.

Of course, especially important are weight elements corresponding to the fundamental weights $\omega=\varpi_{r}$ of $\Phi^{\vee}$. Expressions of the elements $h_{\omega}\left(\varepsilon^{m}\right)$ in terms of semisimple root elements can be easily derived from the expressions of $m \omega$ in terms of the fundamental roots; see [79]. These elements lie in the center of Levi factors of maximal parabolic subgroups. In the usual Chevalley groups over small fields there may be no nontrivial weight elements for a given fundamental weight. However, in the extended simply connected Chevalley groups they always form a one-parameter subgroup isomorphic to the multiplicative group $K^{*}$ of the ground field $K$. The conjugates of such one-parameter subgroups under the action of the Weyl group very often generate the whole extended split maximal torus $\bar{T}_{\mathrm{sc}}$, at least up to a central factor. In Proposition 1 we show that for this it is necessary that $\omega$ generate the lattice $P\left(\Phi^{\vee}\right)$ over the lattice $Q\left(\Phi^{\vee}\right)$.

The elements $h_{\omega}(\varepsilon)$ have a particularly transparent structure when $\omega$ is a microweight of $\Phi^{\vee}$, "poids minuscule" in the sense of $\mathrm{N}$. Bourbaki 4]. We refer to [76, 56, 58, 80, for a thorough discussion of the relevance of microweights and the corresponding bibliography. Recall that the following weights are microweights: all fundamental weights for $\mathrm{A}_{l} ; \varpi_{l}$ for $\mathrm{B}_{l} ; \varpi_{1}$ for $\mathrm{C}_{l} ; \varpi_{1}, \varpi_{l-1}$ and $\varpi_{l}$ for $\mathrm{D}_{l} ; \varpi_{1}$ and $\varpi_{6}$ for $\mathrm{E}_{6}$; and $\varpi_{7}$ for $\mathrm{E}_{7}$. The systems of types $\mathrm{E}_{8}, \mathrm{~F}_{4}, \mathrm{G}_{2}$ have no microweights. A weight $\omega$ of $\Phi^{\vee}$ is a microweight precisely when the unipotent radical of the corresponding parabolic subgroup in $G$ is Abelian, or, what is the same, when the set $\Sigma_{\omega}=\{\alpha \in \Phi \mid(\alpha, \omega)>0\}$ is Abelian in the sense that 
the sum of any two of its roots is not a root. Weight elements of type $\omega$, where $\omega$ is a microweight of $\Phi^{\vee}$, will be called microweight elements.

We are primarily interested in the Bruhat decomposition of microweight elements. Let $U$ and $U^{-}$denote the subgroups of $G$ spanned by all unipotent root subgroups $X_{\alpha}$, where $\alpha \in \Phi^{+}$or, respectively, $\alpha \in \Phi^{-}$. Let $\bar{B}=U \bar{T}$ be the standard Borel subgroup of the extended 1 simply connected Chevalley group $\bar{G}=\bar{G}_{\mathrm{sc}}(\Phi, K)$. Next, let $\bar{N}=\bar{N}(\Phi, K)$ be the subgroup of $\bar{G}$ generated by $\bar{T}$ and all $w_{\alpha}(1), \alpha \in \Phi$; see [3, 36, 43. Almost always $\bar{N}$ coincides with the normalizer of $\bar{T}$ in $\bar{G}$. The quotient group $\bar{N} / \bar{T}$ is isomorphic to the Weyl group $W=W(\Phi)$ of the root system $\Phi$. As usual, we denote by $w_{\gamma}$ the reflection with respect to a root $\gamma \in \Phi$. For an element $w \in W$ we denote by $n_{w}$ its preimage in $\bar{N}$. For example, one can take $n_{w_{\alpha}}=w_{\alpha}(1)$. In general, we tend to choose our $n_{w}$ from the extended Weyl group $\widetilde{W}(\Phi)$, i.e., the subgroup of $N$ generated by all $w_{\alpha}(1), \alpha \in \Phi$; see 75. When no confusion may possibly arise, we simply write $w$ instead of $n_{w}$. Then the Bruhat decomposition for $\bar{G}$ asserts that $\bar{G}$ may be written as a disjoint union of Borel double cosets $\bar{B} w \bar{B}, w \in W$. We denote by $m=m(\omega)$ the maximal number of pairwise orthogonal long roots contained in the set $\Sigma_{\omega}=\{\alpha \in \Sigma \mid(\alpha, \omega)>0\}$.

Theorem 1. Let $\omega$ be a microweight of the root system $\Phi^{\vee}$. Suppose that char $K \neq 2$ if $\Phi=\mathrm{B}_{l}, \mathrm{C}_{l}$. Then any weight element $x h_{\omega}(\varepsilon) x^{-1}, x \in \bar{G}, \varepsilon \in K^{*}$, of type $\omega$ belongs to one of the Bruhat cosets

$$
\bar{B} w \bar{B}=\bar{B} w_{\gamma_{1}} \cdots w_{\gamma_{r+s}} \bar{B}
$$

where $\gamma_{1}, \ldots, \gamma_{r+s}$ are pairwise strictly orthogonal roots. Moreover, if $\gamma_{1}, \ldots, \gamma_{r}$ are long and $\gamma_{r+1}, \ldots, \gamma_{r+s}$ are short, then $r+2 s \leq m(\omega)$. For a fixed $x \in \bar{G}$, the element $w$ in the Bruhat decomposition of $x h_{\omega}(\varepsilon) x^{-1}$ does not depend on the choice of $\varepsilon \neq 0,1$.

Recall that two roots $\alpha, \beta$ are said to be strictly orthogonal if $\alpha \pm \beta$ are not roots (see [4]). Any two orthogonal long roots are strictly orthogonal, but this is not necessarily true for short roots. Below we list the values of $m$ for various microweights (see [10], compare also the table at the end of [62], §2).

\section{TABle 1}

$\begin{array}{lll}\mathrm{A}_{l} & \omega=\varpi_{k} & m=\min (k, l+1-k) \\ \mathrm{B}_{l} & \omega=\varpi_{1} & m=2 \\ \mathrm{C}_{l} & \omega=\varpi_{l} & m=l \\ \mathrm{D}_{l} & \omega=\varpi_{1} & m=2 \\ & \omega=\varpi_{l-1}, \varpi_{l} & m=[l / 2] \\ \mathrm{E}_{6} & \omega=\varpi_{1}, \varpi_{6} & m=2 \\ \mathrm{E}_{7} & \omega=\varpi_{7} & m=3\end{array}$

We recall that here $\omega$ denotes a weight of the dual root system - the list in N. Bourbaki [5. Chapter VII, $\$ 7.4]$ obviously contains two mistakes for $\mathrm{B}_{l}$ and $\mathrm{C}_{l}$ - precisely because each author attempted to take duality into account. But the number of odd authors turned out to be even 2

\footnotetext{
${ }^{1}$ The reader who is not willing to think in terms of extended Chevalley groups may henceforth assume that we always consider adjoint Chevalley groups — as defined in [36]! - and remove bars everywhere, putting the subscript ad instead. All results make as much sense in this setting as they do for the extended simply connected Chevalley groups. However, the author feels much more comfortable with $\mathrm{GL}_{n}$ than he does with $\mathrm{PGL}_{n}$.

${ }^{2}$ The list in [4 Exercise 6.4.15] is correct. One can assume that the main text and the exercises were compiled by different individuals.
} 
Actually, the result could have been stated in a much more precise form. Namely, we could derive a lot of information concerning Borel factors as well, or rather the factors $u=u(\varepsilon), v=v(\varepsilon)$, in the unipotent radical $U$, as we collect together all diagonal factors, $x h_{\omega}(\varepsilon) x^{-1}=d u w v$. It turns out that not merely one factor, as in the general case, but both of them may be chosen in the subgroup $U_{w}^{-}=U \cap w^{-1} U^{-} w$. Furthermore, for an appropriate ordering of positive roots, most of the coefficients $u_{\alpha}, v_{\alpha}$ in the expansions $u=\prod x_{\alpha}\left(u_{\alpha}\right), v=\prod x_{\alpha}\left(v_{\alpha}\right)$ do not depend on the choice of $\varepsilon \neq 0,1$ and, moreover, $u_{\alpha}=-v_{\alpha}$. The remaining coefficients $u_{\alpha}(\varepsilon)$ and $v_{\alpha}(\varepsilon)$ are rational functions in $\varepsilon$, with numerators and denominators of degree at most one. This additional information is requisite to apply our theorem to the description of subgroups in $\bar{G}$ containing the split maximal torus $\bar{T}$. We intend to return to these issues in one of our joint papers with Vladimir Nesterov.

The paper is organized as follows. In $\S 1$ we discuss the general context and some related results. In $\S 2$ we recall basic notation pertaining to Chevalley groups. In $\S \S 3-5$ we discuss the definition of weight elements and elucidate it in the classical examples. In $\S 6$ we recall the Berman-Moody construction, while in $\S 7$ we calculate the action of weight elements in irreducible representations. In $\S 8$ we answer the question as to when weight elements of a given type generate the whole split maximal torus, and in $\S 9$ we completely determine which weight elements are induced by conjugation on regularly embedded subgroups. In $\S \S 10-12$ we study Borel orbits of Levi factors for the case of parabolic subgroups with Abelian unipotent radical. In $\S 13$ we prove a result on the typical Bruhat decomposition of a weight element. Finally, in $\S 14$ we prove Theorem 1, and in $\$ 15$ we state some open problems. Part of the content of the present paper was announced without proofs in [10, 14, 16, while most of the proofs circulated in the form of a preprint; see [7].

\section{$\S 1$. BACKGROUND AND HISTORY}

This theorem was conceived in the 1980s, out of the desire to extend to all Chevalley groups the approach towards classification of overgroups of split maximal tori, proposed for the general linear groups in the work by Zenon Borewicz and the author; see [1, 2, 6]. Namely, the proofs in those papers were based on the study of groups generated by two conjugates of a one-parameter group of pseudoreflections $\left\{d_{i}(\varepsilon), \varepsilon \in K^{*}\right\}$. The methods of those papers were definitely construed in [9, 55].

In the terminology of the present paper, a pseudoreflection in the general linear group is nothing else but a microweight element corresponding to the first fundamental weight $\varpi_{1}$ of the simply connected Chevalley group of type $A_{l}$, in the fundamental representation of highest weight $\varpi_{1}$. In [7, 9, the proofs from the papers [1, 2, 6] were recast so as to avoid any reference to matrices whatsoever, and effectively expressed in terms of Bruhat decomposition. In particular, those papers contained the simplest case of the above theorem, namely that of pseudoreflections in the general linear group.

In full generality this theorem was first announced in 10, but a detailed proof has never been published. It asserts that, for all types, the microweight elements behave in the best possible way and that - apart from the case $\mathrm{C}_{l}$ - one can always find small microweight elements. This was instrumental in the proof of analogs of results from [1, 2, 6] for other extended Chevalley groups. The case of the general symplectic group is exceptional. Its analysis in the paper [20] by the author and Elizaveta Dybkova was from the very start based on the analysis of semisimple root elements 3 the elements $h_{\varpi_{l}}(\varepsilon)$ were only used at the very last moment. In [11, 16 and 17] we used this theorem in

\footnotetext{
${ }^{3}$ Because of duality between $\mathrm{C}_{l}$ and $\mathrm{B}_{l}$, the semisimple long root elements of the symplectic group behave practically as microweight elements; compare with [31] 32 .
} 
the proof of conjugacy and classification theorems for subgroups of extended Chevalley groups containing a split maximal torus. A general overview of this topic and many further references can be found in [26, 28, 15, 16, 78, 79, 50,

Subgroups of finite Chevalley groups containing a split maximal torus were described by Gary Seitz [69]. Generalizations of his results to infinite fields (see [16, 78]) require more complicated semisimple elements than the microweight ones. As a matter of fact, the groups of types $\mathrm{E}_{8}, \mathrm{~F}_{4}$, and $\mathrm{G}_{2}$ have no microweights at all. But even for the special linear group $\mathrm{SL}(n, K)$ the use of microweights leads to somewhat exaggerated lower bounds on the cardinality $|K|$; see $[8$. Next to the microweight elements, the nicest semisimple elements are semisimple long root elements. They were studied in a similar context by the author and Andrei Semenov [12, 13, 14, 22, 23, 31, 32, 71]; see below.

From a technical viewpoint, the above theorem amounts to determining Borel orbits for the Levi factor $L_{Q}$ of a parabolic subgroup $Q$ with Abelian unipotent radical $U_{Q}$ in its action on the radical $U_{Q}$ by conjugation. Thus, the present paper has a nontrivial intersection with the paper 62 by R. Richardson, G. Röhrle, and R. Steinberg, where explicit representatives of the $L_{Q}$-orbits on $U_{Q}$ were determined. However, our results are more general than Theorem 1.2 of 62 in the following two respects. First, we determine orbits of the Borel subgroup $B_{Q}$ of the Levi factor $L_{Q}$, rather than those of $L_{Q}$ itself. Second, we do not assume our ground field $K$ to be algebraically closed. As a result, our answer is somewhat more complicated than that in 62. First, since we are interested in Borel orbits, we cannot restrict ourselves to the subsequences of a maximal system of orthogonal long roots. Instead, we need to consider all such sequences, and short roots must also be taken into account. Second, since our field is not algebraically closed, we cannot simultaneously make all of the coefficients to be equal to 1 in the final answer: some of the coefficients have to run over representatives of $K^{*}$ modulo $K^{* 2}$.

One may note that all representations induced by the conjugation actions of $L_{Q}$ on $U_{Q}$ are small-dimensional. At least for the case where char $K=0$, the orbits of $L_{Q}$ itself have been well known for some 40 years. The earliest reference for the 27-dimensional representation of $L_{Q} \cong \bar{G}\left(\mathrm{E}_{6}, K\right)$, which appears as a Levi factor in $G\left(\mathrm{E}_{7}, K\right)$, is perhaps [53. Notice that this work describes the orbits of the extended Chevalley group of type $\mathrm{E}_{6}$. In this case there are four such orbits: 0 , white, grey, and black vectors; see [46, 76]. Observe that the usual group $G\left(\mathrm{E}_{6}, K\right)$ may have infinitely many orbits if $K$ is not algebraically closed.

All other Levi factors arising in the proof of the theorem are classical. For them the corresponding orbits must have been enumerated even before that. At least for algebraically closed fields of characteristic 0, Borel orbits in (not necessarily) linear actions of reductive groups have been intensively studied by A. Ėlashvili, V. Popov, M. Brion and many others (see references in [41, 30, 27]). A very vivid description of some classical cases can be found in the papers of W. Hesselink and H. Bürgstein [52, 42. For some representations of exceptional groups, in particular for the representations appearing in the above theorem, Borel orbits were calculated around 1984, independently and using different methods, by A. Èlashvili and the present author. I can recall no good reason why these results have never been published.

To calculate the Bruhat decomposition of other weight elements, one must consider Borel orbits of Levi subgroups in the unipotent radicals (or, rather, consecutive quotients of the lower central series of the unipotent radicals) for other parabolic subgroups. These quotients and the corresponding representations (the so-called internal Chevalley modules) have been intensively studied by many authors, notably by Röhrle and Popov [39, 59, 60, 61, 62, 63, 64, 65, 66, 67, 68. We do not try to attach a complete bibliography concerning the geometry of such actions, modality calculations, etc. 
The next case after the parabolic subgroups with Abelian unipotent radical is that of the special unipotent radical, where the center coincides with the commutator and is isomorphic to the additive group $K^{+}$. The corresponding weight elements are the usual semisimple long root elements. The Bruhat decomposition of such elements has been studied by the author and Semenov [12, 14, 22, 23, 31, 32, 71]. For this case, it is no longer true that all elements $x h_{\alpha}(\varepsilon) x^{-1}, \varepsilon \neq 0,1$, lie in the same Bruhat double coset. However, all of them lie in the $\operatorname{cosets} \bar{B} w \bar{B}$ coming from some subsystem $\Delta \subseteq \Phi$ which is a subsystem of $\mathrm{D}_{4}$, possibly twisted. A preliminary form of this reduction can be found in [12, 14, whereas in [22, 23, 71, a more precise statement referring to the long root tori was established in full generality. This reduction to $\mathrm{D}_{4}$ was rediscovered by Gerhard Röhrle 65. Of course, here again the orbits of the Levi factor $L_{Q}$ itself on the quotient group of $U_{Q}$ modulo the center might have been determined long ago - at least in characteristic 0 . The senior case that occurred here is that of the 56-dimensional representations of $L_{Q} \cong \bar{G}\left(\mathrm{E}_{7}, K\right)$, arising in a parabolic subgroup of $G\left(\mathrm{E}_{8}, K\right)$. For this case the orbits were classified in [51. Of course, again to study Bruhat decomposition we need to calculate Borel orbits.

The only other case which has been understood completely is that of weight elements in the general linear group $\operatorname{GL}(n, K)$. Actually, in this case it is relatively easy to describe all elements arising in the Bruhat decomposition of any $m$-dimensional transformation, i.e., of an element $x \in \operatorname{GL}(n, K)$ such that $\operatorname{rk}(x-e)=m$. The case of $m=1$, which includes microweight elements of type $\varpi_{1}$ and unipotent root elements, was considered in [9]. The case where $m=2$, which includes, for example, semisimple long root elements of the special linear and the symplectic groups and weight elements of type $\varpi_{1}$ in orthogonal groups, was considered in [13, 31]. In the recent paper [48, by Erich Ellers and Nikolai Gordeev, one can find similar results for the elements of large residue.

\section{§2. Chevalley Groups}

In this section we describe the basic notation and some requisite facts concerning Chevalley groups; see [3, 5, 34, 35, 43.

$\mathbf{1}^{\circ}$. Chevalley groups. Let $\Phi$ be a reduced root system of rank $l$, and let $P$ be a lattice lying between the root lattice $Q(\Phi)$ and the weight lattice $P(\Phi)$. We denote by $G_{P}(\Phi, K)$ the Chevalley group of type $(\Phi, P)$ over $K$, and $T=T_{P}(\Phi, K)$ is a fixed split maximal torus. When $P$ does not have any particular role, we omit it in the notation.

The usual constructions of Chevalley groups are based on the fact that a Chevalley base can be chosen in a complex semisimple Lie algebra $L=L_{\mathbb{C}}$ of type $\Phi$. Fix an order on $\Phi$, with $\Phi^{+}, \Phi^{-}$, and $\Pi=\left\{\alpha_{1}, \ldots, \alpha_{l}\right\}$ being the sets of positive, negative, and fundamental roots, respectively. A Chevalley base $\left\{e_{\alpha}, \alpha \in \Phi ; h_{\alpha}, \alpha \in \Pi\right\}$ is a normalized Weyl base such that all structure constants are integers. The integral lattice $L_{\mathbb{Z}}$ spanned by a Chevalley base is called an admissible $\mathbb{Z}$-form or a Chevalley order in $L$. The easiest way to construct the adjoint Chevalley group $G_{\text {ad }}(\Phi, K)$ over a field $K$ is to regard it as an automorphism group of the Chevalley algebra $L_{K}=L_{\mathbb{Z}} \otimes K$ generated by certain unipotent and semisimple automorphisms [3, 36, 43, 70. However, the necessity to invoke semisimple automorphisms is often forgotten, which leads to incessant mistakes.

Now, let $\pi: L \rightarrow \operatorname{gl}(V)$ be a representation of the complex semisimple Lie algebra $L=L_{\mathbb{C}}$ in a finite-dimensional vector space $V$ over $\mathbb{C}$. We often omit the symbol $\pi$ when denoting the action of $G$ on $V$, and for $x \in L$ and $u \in V$ we simply write $x u$ instead of $\pi(x) u$. A lattice $V_{\mathbb{Z}}$ in $V$ is said to be admissible with respect to $L_{\mathbb{Z}}$ if it is invariant under the action of the divided powers $e^{(m)} \alpha=e_{\alpha}^{m} / m$ !, in other words, if $e_{\alpha}^{(m)} V_{\mathbb{Z}} \subseteq V_{\mathbb{Z}}$ 
for all $\alpha \in \Phi, m \in \mathbb{Z}^{+}$. It is well known that such a lattice always exists and is the direct sum of its weight components [3, 34, 35].

Set $x_{\alpha}(\xi)=x_{\alpha}^{\pi}(\xi)=\exp \left(\xi \pi\left(e_{\alpha}\right)\right)$, where $\alpha \in \Phi, \xi \in R$, and exp is defined by the usual formula. By the very definition of an admissible lattice, the elements $x_{\alpha}(\xi), \alpha \in \Phi$, $\xi \in R$, stabilize the $R$-module $V_{R}=V_{\mathbb{Z}} \otimes_{\mathbb{Z}} R$. Here $\xi^{m}$ acts on the second component while $e_{\alpha}^{(m)}$ acts on the first. The group

$$
\mathrm{E}_{\pi}(\Phi, R)=\left\langle x_{\alpha}(\xi), \alpha \in \Phi, \xi \in R\right\rangle \leq \mathrm{GL}\left(V_{R}\right)
$$

is called the elementary Chevalley group of type $\Phi$ over $R$ in the representation $\pi$.

Over a field $K$, the Chevalley group $G_{\pi}(\Phi, K)$ is generated by $E_{\pi}(\Phi, K)$ and certain semisimple elements. In many important cases, for example, when $K$ is algebraically closed, or when $G_{\pi}=G_{\mathrm{sc}}$ is simply connected, we have $G_{\pi}(\Phi, K)=E_{\pi}(\Phi, K)$.

Thus, if the lattice of weights $P(\pi)$ of the representation $\pi$ equals $P$, then from the very start the groups $E_{P}(\Phi, K)$ and $G_{P}(\Phi, K)$ arise together with their representation on the vector space $V_{K}=V_{\mathbb{Z}} \otimes K$, which we denote by the same letter $\pi$.

$2^{\mathbf{o}}$. Root elements. With any root $\alpha \in \Phi$, one can associate unipotent root elements $x_{\alpha}(\xi), \xi \in K$. If we wish to stress that these root unipotents correspond to a given choice of a split maximal torus, we call them elementary root unipotents. In general, any conjugate of an elementary root unipotent is called a root unipotent. A root unipotent $g x_{\alpha}(\xi) g^{-1}$ is long or short depending on whether the root $\alpha$ is long or short.

Now, suppose $\alpha \in \Phi$ and $\varepsilon \in K^{*}$. As usual, we set $h_{\alpha}(\varepsilon)=w_{\alpha}(\varepsilon) w_{\alpha}(1)^{-1}$, where $w_{\alpha}(\varepsilon)=x_{\alpha}(\varepsilon) x_{-\alpha}\left(-\varepsilon^{-1}\right) x_{\alpha}(\varepsilon)$. The elements $h_{\alpha}(\varepsilon)$ and their conjugates are semisimple root elements. They are called long or short depending on whether the root $\alpha$ is long or short.

Most calculations in Chevalley groups are based on the familiar Steinberg relations among the elements $x_{\alpha}(\xi), w_{\alpha}(\varepsilon), h_{\alpha}(\varepsilon)$. The most important among these relations are the additivity of $x_{\alpha}(\xi)$ in $\xi$, the multiplicativity of $h_{\alpha}(\varepsilon)$ in $\varepsilon$, and the Chevalley commutator formula, which asserts that

$$
\left[x_{\alpha}(\xi), x_{\beta}(\eta)\right]=\prod x_{i \alpha+j \beta}\left(N_{\alpha \beta i j} \xi^{i} \eta^{j}\right)
$$

for any $\alpha, \beta \in \Phi$ with $\alpha+\beta \neq 0$ and any $\xi, \eta \in R$.

Here for two elements $x, y$ of a group $G$ we denote by $[x, y]$ their commutator $x y x^{-1} y^{-1}$, whereas the product on the right-hand side is taken over all roots of the form $i \alpha+j \beta \in \Phi$, $i, j \in \mathbb{N}$, in a fixed order. The constants $N_{\alpha \beta i j}$ do not depend on $\xi$ and $\eta$, but, in general, they may depend on the order. The integers $N_{\alpha \beta i j}$ are called the structure constants of the Chevalley group; we refer the reader to [43, 72, 76, 81, 82] for the details and further references.

For a fixed $\alpha \in \Phi$, the map $x_{\alpha}: \xi \mapsto x_{\alpha}(\xi)$ is a homomorphism of the additive group $R^{+}$of $R$ to the one-parameter subgroup $X_{\alpha}=\left\{x_{\alpha}(\xi) \mid \xi \in R\right\}$, which is called the elementary unipotent root subgroup corresponding to $\alpha$. In fact, $x_{\alpha}$ is an isomorphism of $R^{+}$onto $X_{\alpha}$. When it does not lead to confusion, we omit the specifications elementary and unipotent and simply speak of root elements and root subgroups.

$3^{\text {o }}$. Bruhat decomposition. Now, let $N=N(\Phi, K)$ be the subgroup in $G$ generated by $T=T(\Phi, K)$ and all elements $w_{\alpha}(1), \alpha \in \Phi$. It is well known that for $|K| \geq 4$ the group $N$ coincides with the normalizer of $T$ in $G$; see 34]. The quotient group $N / T$ is canonically isomorphic to the Weyl group $W$, and for each $w \in W$ we fix a preimage $n_{w}$ of $w$ in $N$. Usually, when we speak of subgroups containing $T$, we identify $w$ and $n_{w}$. For instance, we write $B w B$ instead of $B n_{w} B$ to denote the double Borel cosets. 
Set

$$
\begin{gathered}
U=U(\Phi, K)=\left\langle x_{\alpha}(\xi), \alpha \in \Phi^{+}, \xi \in K\right\rangle, \\
U^{-}=U^{-}(\Phi, K)=\left\langle x_{\alpha}(\xi), \alpha \in \Phi^{-}, \xi \in K\right\rangle .
\end{gathered}
$$

Then $U=U(\Phi, K)$ is the product of elementary root subgroups $X_{\alpha}, \alpha \in \Phi^{+}$, in any fixed order. In other words, any element $u \in U$ may be written in the form $u=\prod x_{\alpha}\left(u_{\alpha}\right)$, where $\alpha$ runs over the set $\Phi^{+}$of positive roots and the coefficients $u_{\alpha} \in K$ are uniquely determined by $u$ itself and by the order on $\Phi^{+}$. The product $B=B(\Phi, K)$ of the groups $T$ and $U$ is called the standard Borel subgroup of $G$ corresponding to a given choice of $T$ and $\Phi^{+}$. The group $U$ is called the unipotent radical of $B$. The product $B^{-}=B^{-}(\Phi, R)$ of $T$ and $U^{-}$is called the standard Borel subgroup opposite to $B$.

The Bruhat lemma asserts that the $n_{w}, w \in W$, form a system of double coset representatives for $B$ in $G$. In other words, any element $x$ of $G=G(\Phi, K)$ can be written as $x=b_{1} n_{w} b_{2}$, where $b_{1}, b_{2} \in B$ and $w$ is uniquely determined by $x$. This decomposition of $x$ is referred to as its Bruhat decomposition.

$4^{\mathrm{o}}$. Standard subgroups. The groups $U$ and $U^{-}$considered in the preceding subsection are special cases of the groups $E(S)=E(S, R)$, which can be ascribed to any closed subset $S$ in $\Phi$. Recall that a subset $S$ in $\Phi$ is said to be closed if for any two roots $\alpha, \beta \in S$ such that $\alpha+\beta \in \Phi$ we have $\alpha+\beta \in S$. Define $E(S)=E(S, R)$ as the subgroup generated by all elementary root subgroups $X_{\alpha}, \alpha \in S$,

$$
E(S, R)=\left\langle x_{\alpha}(\xi), \alpha \in S, \xi \in R\right\rangle .
$$

In this notation, $U$ and $U^{-}$coincide with $E\left(\Phi^{+}\right)$and $E\left(\Phi^{-}\right)$, respectively. The groups $E(S)$ are particularly important when the set $S$ is special alias unipotent, in other words, $S \cap(-S)=\varnothing$. In this case $E(S)$ is precisely the product of all $X_{\alpha}, \alpha \in S$, in any fixed order. Set $G(S)=T(\Phi) E(S)$.

Again let $S \subseteq \Phi$ be any closed set of roots. Then $S$ is the disjoint union of its reductive alias symmetric part $S^{r}$, which consists of $\alpha \in S$ such that $-\alpha \in S$, and its unipotent part $S^{u}$, which consists of $\alpha \in S$ such that $-\alpha \notin S$. The set $S^{r}$ is a closed subsystem of $\Phi$ whereas the set $S^{u}$ is special. Moreover, $S^{u}$ is an ideal in $S$; in other words, if $\alpha \in S$, $\beta \in S^{u}$, and $\alpha+\beta \in \Phi$, then $\alpha+\beta \in S^{u}$. We avoid the common notation $S^{+}$and $S^{-}$, preferring to reserve them for $S^{+}=S \cap \Phi^{+}$and $S^{-}=S \cap \Phi^{-}$. It is easily seen that the group $G(S)$ is the semidirect product of the reductive subgroup $G\left(S^{r}\right)$, which is called a Levi subgroup of $G(S)$ ), and the unipotent subgroup $E\left(S^{u}\right)$, which in its turn is called the unipotent radical of $G(S)$. Similarly, the group $E(S)$ is the semidirect product of $E\left(S^{r}\right)$ and $E\left(S^{u}\right)$.

Two sets of roots $S_{1}, S_{2} \subseteq \Phi$ are said to be conjugate if there exists an element $w$ of the Weyl group $W=\mathrm{W}(\Phi)$ such that $w S_{1}=S_{2}$. For conjugate sets $S_{1}$ and $S_{2}$, there exists $n \in \widetilde{W}(\Phi)$ such that $n G\left(S_{1}\right) n^{-1}=G\left(S_{2}\right)$. We say that an element $w \in W$ normalizes $S$ if $w S=S$. The set $X(S)$ of all $w \in W$ that normalize $S$ is called the Weyl normalizer of $S$. It is clear that $X(S)$ contains the Weyl subgroup $W(S)=W\left(S^{r}\right)$. Furthermore, if $S=S^{r}$ is a root subsystem, then $X(S)$ coincides with the normalizer of $W(S)$ in $W$; see [43. Denote by $N(S)$ the subgroup of $G$ generated by $G(S)$ and $n_{w}, w \in X(S)$. A theorem of Tits 74 implies that almost always $N(S)$ coincides with the normalizer of $G(S)$ in $G$. The only cases where this identity may fail are those of the fields $K=\mathbb{F}_{2}$, $\mathbb{F}_{3}$, and even over those fields it only fails for some $\Phi$ and $S$.

$5^{\circ}$. Parabolic subgroups. Recall that we have fixed an order on the root system, which determines $\Pi, \Phi^{+}$, and $\Phi^{-}$. A standard parabolic subset $P$ is a closed set of roots containing $\Phi^{+}$. A parabolic subset $Q$ is a subset conjugate to a standard parabolic 
one. It is well known that the parabolic subsets fall into $2^{l}$ conjugacy classes, where $l=\operatorname{rk}(\Phi)$ is the rank of $\Phi$. The standard parabolic subsets are pairwise nonconjugate and correspond bijectively to the subsets $J \subseteq \Pi$ of the fundamental system. Namely, for a subset $J \subseteq \Pi$ we define $P_{J}$ to be the smallest closed set of roots containing $\Phi^{+}$and $-J$. The most important parabolic subsets are the maximal ones. A maximal parabolic subset corresponds to a set $J=J_{r}, 1 \leq r \leq l$, that contains all fundamental roots apart from $\alpha_{r}$. The corresponding parabolic set $P_{J_{r}}$ is maximal among the closed subsets and will be denoted simply by $P_{r}$. Thus, there are precisely $l$ conjugacy classes of maximal parabolic subsets.

The standard parabolic subgroups of a Chevalley group $G$ are subgroups containing the standard Borel subgroup $B$. In turn, the parabolic subgroups are conjugates of the standard parabolic ones. A classical theorem of Tits asserts that the map $P_{J} \mapsto G\left(P_{J}\right)$ is a bijection between the set of standard parabolic subsets and the set of standard parabolic subgroups. For a parabolic subset $P$, we denote by $U_{P}$ and $L_{P}$ the unipotent radical and (the standard) Levi subgroup of the parabolic subgroup $G(P)$, respectively. For a maximal parabolic subset $P=P_{r}$ we simply write $U_{P}=U_{r}$ and $L_{P}=L_{r}$.

$6^{\mathbf{o}}$. Root subsystems. Subsystems of a root system were classified by Borel-de Siebenthal and Dynkin 25] with the help of the following remarkable construction. The resulting tables and many further references can be found in [44, 24] and in [50].

Let $\bar{\Pi}=\Pi \cup\left\{\alpha_{0}\right\}$ be the extended fundamental system, obtained by adjoining to $\Pi$ the root $\alpha_{0}=-\delta$, where $\delta$ is the maximal root with respect to $\Pi$. Let $\Delta_{r}, 1 \leq r \leq l$, be the smallest closed set of roots containing all roots $\pm \alpha$, where $\alpha \in \bar{\Pi} \backslash\left\{\alpha_{r}\right\}$. Then $\Delta_{r}$ is a maximal rank subsystem in $\Phi$; in other words, $\operatorname{rk} \Delta_{r}=\operatorname{rk} \Phi$. This subsystem $\Delta_{r}$ may be reducible. It may also coincide with $\Phi$, and this is always the case for $\Phi=\mathrm{A}_{l}$. As the next step we can repeat the same procedure starting with any irreducible component of $\Delta_{r}$, etc. As a result, we get all closed maximal rank subsystems of $\Phi$; see [25. Table 10].

To get all closed root subsystems of $\Phi$, we proceed as follows. Let $\Delta$ be a maximal rank subsystem of $\Phi$ and $\Sigma$ a fundamental root system of $\Delta$. Take an arbitrary subset $J$ in $\Sigma$ and consider the closure of $-J \cup J$. Applying this procedure to all subsets of fundamental roots of maximal rank subsystems, we get all closed root subsystems of $\Phi$ up to conjugacy. For the classical root systems such a classification is obvious; see 25, Table 9]. It turns out that up to conjugacy there are 5 proper subsystems in $\Phi=\mathrm{G}_{2}, 23$ in $\mathrm{F}_{4}, 20$ in $\mathrm{E}_{6}, 46$ in $\mathrm{E}_{7}$, and 76 in $\mathrm{E}_{8}$; see [25, Table 11].

Almost always two isomorphic root subsystems are conjugate. Since later we need the precise answer, we list all exceptions.

- When $\Phi$ is multiply laced, whereas $\Delta$ is simply laced, then, generally speaking, $\Delta$ may be embedded in $\Phi$ in two essentially different ways: at the long roots and at the short roots. To distinguish these embeddings, we write $\widetilde{\Delta} \leq \Phi$ for the short root embedding and $\Delta \leq \Phi$ for the long root embedding.

- In the root systems $\mathrm{B}_{l}$ and $\mathrm{D}_{l}$ we must distinguish $2 \mathrm{~A}_{1}=\left\{ \pm\left(e_{1}-e_{2}\right), \pm\left(e_{3}-e_{4}\right)\right\}$ from $\mathrm{D}_{2}=\left\{ \pm e_{1} \pm e_{2}\right\}$ and $\mathrm{A}_{3}=\left\langle 2 \mathrm{~A}_{1}, e_{2}-e_{3}\right\rangle$ from $\mathrm{D}_{3}=\left\langle\mathrm{D}_{2}, e_{2}-e_{3}\right\rangle$.

- In the root systems $\mathrm{D}_{l}, \mathrm{E}_{7}$, and $\mathrm{E}_{8}$ some root subsystems $\Delta$ of type $\mathrm{A}_{l_{1}}+\cdots+\mathrm{A}_{l_{r}}$, where all $l_{i}$ are odd, fall into two conjugacy classes, which will be denoted by $\Delta^{\prime}$ and $\Delta^{\prime \prime}$, respectively. In the $\mathrm{D}_{l}$ case these two classes are fused by an external automorphism.

\section{§3. Diagonal extensions}

In this section we discuss diagonal automorphisms of Chevalley groups. First, we recall the construction of some semisimple elements that, together with the elementary 
subgroup, generate the whole Chevalley group 4 or even slightly larger groups, see 40 , 10, 12, 14, 16.

Recall that the split maximal torus of the group $G=G_{P}(\Phi, K)$ is isomorphic to the group of $K$-characters of the weight lattice $P$ :

$$
T=T_{P}(\Phi, K) \cong \operatorname{Hom}\left(P, K^{*}\right) .
$$

At the same time, generally speaking, the intersection of $T$ with the elementary subgroup $E=E_{P}(\Phi, K)$, usually denoted by $H=H_{P}(\Phi, K)$, is somewhat smaller:

$$
H=H_{P}(\Phi, K) \cong \operatorname{Hom}\left(P(\Phi), K^{*}\right) / \operatorname{Ker}\left(\operatorname{res}_{P}^{P(\Phi)}\right) .
$$

This means that the elements of $H$ correspond not to all characters of $P$, but rather to the characters that can be extended to the entire weight lattice $P(\Phi)$. Clearly, for the simply connected case we have $T_{\mathrm{sc}}(\Phi, K)=H_{\mathrm{sc}}(\Phi, K)$. Later we think of $P$ as being fixed and suppress it in the notation. Actually, we have

$$
H=H(\Phi, K)=\left\langle h_{\alpha}(\varepsilon), \alpha \in \Phi, \varepsilon \in K^{*}\right\rangle ;
$$

see, e.g., 34. As a matter of fact, not all $h_{\alpha}(\varepsilon)$ 's are needed to generate $H$ : some of them would already suffice. For example, one can take only $h_{\alpha}(\varepsilon)$ 's for the fundamental roots $\alpha \in \Pi$, or only those for the long roots $\alpha \in \Phi_{l}$. For the simply connected case, $H$ is isomorphic to the direct product of $l$ copies of the multiplicative group $K^{*}$. Namely, each element $h \in H_{\mathrm{sc}}$ can be written uniquely in the form $h=h_{\alpha_{1}}\left(\varepsilon_{1}\right) \cdots h_{\alpha_{l}}\left(\varepsilon_{l}\right)$ for some $\varepsilon_{1}, \ldots, \varepsilon_{l} \in K^{*}$. For the adjoint case such a product becomes trivial when $\varepsilon_{1}^{\left(\alpha_{1}, \beta\right)} \cdots \varepsilon_{l}^{\left(\alpha_{l}, \beta\right)}=1$ for all roots $\beta \in \Phi$. The element $h(\chi) \in T(\Phi, K)$ corresponding to a character $\chi \in \operatorname{Hom}\left(P, K^{*}\right)$ acts on the elementary generators $x_{\alpha}(\xi)$ as follows:

$$
h(\chi) x_{\alpha}(\xi) h(\chi)^{-1}=x_{\alpha}(\chi(\alpha) \xi) .
$$

This formula can be generalized, and an arbitrary $K$-character of the lattice $Q(\Phi)$ - and not merely a character of $P$ - can be taken as $\chi$ in this formula. Such a map realizes a not necessarily internal diagonal automorphism of the group $G$; see [34, 43]. Namely, let $\alpha \mapsto \chi(\alpha)$ be an arbitrary map from the fundamental system $\Pi$ to $K^{*}$. This map can be extended uniquely by linearity up to a homomorphism $Q(\Phi) \rightarrow K^{*}$, i.e., up to a $K$-character of the lattice $Q(\Phi)$ :

$$
\chi\left(m_{1} \alpha_{1}+\cdots+m_{l} \alpha_{l}\right)=\chi\left(\alpha_{1}\right)^{m_{1}} \cdots \chi\left(\alpha_{l}\right)^{m_{l}} .
$$

Lemma 58 in 34] asserts that there exists a unique automorphism $\phi_{\chi}$ of the group $G=$ $G(\Phi, K)$ such that $\phi_{\chi}\left(x_{\alpha}(\xi)\right)=x_{\alpha}(\chi(\alpha) \xi)$ for all $\alpha \in \Phi, \xi \in K$. Such automorphisms are said to be diagonal. Sometimes by diagonal automorphisms one means the cosets of diagonal automorphisms modulo internal diagonal automorphisms. However, we say that an automorphism $\phi$ of $G$ is diagonal if it is constructed by the above procedure, or, what is the same, if $\phi\left(X_{\alpha}\right) \subseteq X_{\alpha}$ for all $\alpha \in \Phi$ and $\phi(h)=h$ for all $h \in H$.

The bulk of diagonal automorphisms are internal. Among all diagonal automorphisms the internal ones are characterized by the fact that they correspond to the $K$-characters of $Q(\Phi)$ that can be extended to characters of the entire weight lattice $P(\Phi)$. This means that, for the simply connected group, the quotient group of diagonal automorphisms modulo the internal ones is isomorphic to the direct product of the quotient groups $K^{*} / K^{* m_{i}}$, where the $m_{i}$ run over all elementary divisors of the finite Abelian group $P(\Phi) / Q(\Phi)$. In particular, when $\Phi=\mathrm{B}_{l}, \mathrm{C}_{l}$, or $\mathrm{D}_{l}$ with $l$ even, or $\Phi=\mathrm{E}_{7}$, all diagonal automorphisms are internal if and only if $K^{*}=K^{* 2}$. For $\Phi=\mathrm{E}_{6}$, the necessary and sufficient condition is that $K^{*}=K^{* 3}$, and when $\Phi=\mathrm{D}_{l}$ with $l$ odd, the necessary and

\footnotetext{
${ }^{4}$ This applies to the case of a ground field. Over a ring there are $K_{1}$-functors and their analogs on top of it.
} 
sufficient condition is that $K^{*}=K^{* 4}$. Sometimes it is convenient to consider extensions of a Chevalley group where all diagonal automorphisms become internal.

There is no need to extend adjoint groups in this manner. It suffices to consider linear operators $h_{\mathrm{ad}}(\chi)$ on the Chevalley algebra that act on a Chevalley base as follows: $h_{\mathrm{ad}}(\chi) h_{i}=h_{i}$ and $h_{\mathrm{ad}}(\chi) x_{\alpha}=\chi(\alpha) x_{\alpha} ;$ see $\left[70,43\right.$. Then the group $T_{\mathrm{ad}}=T_{\mathrm{ad}}(\Phi, K)$, consisting of all such $h(\chi)$ for $\chi \in \operatorname{Hom}\left(Q(\Phi), K^{*}\right)$, normalizes $E_{\mathrm{ad}}$, and the product $G_{\text {ad }}=T_{\text {ad }} E_{\text {ad }}$ is precisely the adjoint Chevalley group of type $\Phi$ over $K$. In [70] one can find identification of these groups for the classical series.

These extended groups are precisely the groups considered by Chevalley himself in his original paper 36. Also, they are precisely the groups of points of the adjoint ChevalleyDemazure group schemes. No wonder, the main objective of Chevalley was exactly to construct semisimple algebraic groups. For an expert in algebraic groups, the adjoint group of type $\mathrm{A}_{l}$ is $\mathrm{PGL}_{l+1}$, rather than $\mathrm{PSL}_{l+1}$, as (preposterously!) presumed by laymen. Later, the interest in finite simple groups shifted attention to the elementary Chevalley groups to such an extent that in 34 they were even - erroneously!!! — called Chevalley groups. Thus, in the terminology of [34, the adjoint Chevalley group of type $\mathrm{A}_{l}$ is $\mathrm{PSL}_{l+1}$; see the table at the end of $\S 3$ therein. One need not go far to observe the resulting devastating confusion; it suffices to compare this with the items of the same table, identifying groups of types $\mathrm{B}_{l}$ and $\mathrm{D}_{l}$ !

It is somewhat more difficult to construct such an extension for the simply connected case because here, to keep the maximal torus connected, one must increase its dimension by one (or by two when the universal center is not cyclic). An elementary construction of such an extension was only given in 1975 in [40, and we recall this construction in $\S 6$. As examples of extended simply connected Chevalley groups, one may think of GL $(n, K)$ for type $\mathrm{A}_{l}$ and of $\operatorname{GSp}(2 l, K)$ for type $\mathrm{C}_{l}$.

\section{$\S 4$. Weight ELEMENTS}

Recall that a semisimple root element $h_{\alpha}(\varepsilon)$ acts on unipotent root elements $x_{\beta}(\xi)$ as follows:

$$
h_{\alpha}(\varepsilon) x_{\beta}(\xi) h_{\alpha}(\varepsilon)^{-1}=x_{\beta}\left(\varepsilon^{\langle\beta, \alpha\rangle} \xi\right) .
$$

Here $\langle\beta, \alpha\rangle=2(\beta, \alpha) /(\alpha, \alpha)=\left(\beta, \alpha^{\vee}\right)$ is the corresponding Cartan number, while $\alpha^{\vee}=$ $2 \alpha /(\alpha, \alpha)$ is the dual root of $\alpha$. Thus, conjugation by $h_{\alpha}(\varepsilon)$ realizes an internal diagonal automorphism on $G$ that corresponds to the $K$-character $\chi=\chi_{\alpha, \varepsilon}$ specified by

$$
\chi_{\alpha, \varepsilon}(\beta)=\varepsilon^{\langle\beta, \alpha\rangle}=\varepsilon^{\left(\beta, \alpha^{\vee}\right)} .
$$

Since $\left(\beta, \alpha^{\vee}\right)=\langle\beta, \alpha\rangle \in \mathbb{Z}$, this character is well defined. By definition, $(\beta, \omega) \in \mathbb{Z}$ for any $\beta \in \Phi$ and any $\omega \in P\left(\Phi^{\vee}\right)$, where $\Phi^{\vee}$ is the dual root system, $\Phi^{\vee}=\left\{\alpha^{\vee} \mid \alpha \in \Phi\right\}$. Thus, for any $\omega \in P\left(\Phi^{\vee}\right)$ and any $\varepsilon \in K^{*}$ we can define the $K$-character $\chi_{\omega, \varepsilon} \in \operatorname{Hom}\left(Q(\Phi), K^{*}\right)$ by the formula

$$
\chi_{\omega, \varepsilon}(\beta)=\varepsilon^{(\beta, \omega)} .
$$

This character is linear in $\omega$ :

$$
\chi_{m_{1} \omega_{1}+m_{2} \omega_{2}, \varepsilon}=\chi_{\omega_{1}, \varepsilon}^{m_{1}} \chi_{\omega_{2}, \varepsilon}^{m_{2}} .
$$

Furthermore, $\chi_{m \omega, \varepsilon}=\chi_{\omega, \varepsilon^{m}}$. Now we can define a weight element $h_{\omega}(\varepsilon)$ as an element such that the conjugation by $h_{\omega}(\varepsilon)$ furnishes the diagonal automorphism of $G=G(\Phi, K)$ corresponding to the character $\chi_{\omega, \varepsilon}$ :

$$
h_{\omega}(\varepsilon) x_{\beta}(\xi) h_{\omega}(\varepsilon)^{-1}=x_{\beta}\left(\varepsilon^{(\beta, \omega)} \xi\right) .
$$

Clearly, this formula implies that $h_{\omega}(\varepsilon)$ commutes with all $h_{\alpha}(\eta)$. Indeed, we have

$$
h_{\omega}(\varepsilon) w_{\beta}(\eta) h_{\omega}(\varepsilon)^{-1}=x_{\beta}\left(\varepsilon^{(\beta, \omega)} \eta\right) x_{-\beta}\left(\varepsilon^{(-\beta, \omega)} \eta^{-1}\right) x_{\beta}\left(\varepsilon^{(\beta, \omega)} \eta\right)=w_{\beta}\left(\varepsilon^{(\beta, \omega)} \eta\right),
$$


whence

$$
\begin{aligned}
h_{\omega}(\varepsilon) h_{\beta}(\eta) h_{\omega}(\varepsilon)^{-1} & =h_{\omega}(\varepsilon) w_{\beta}(\eta) w_{\beta}(-1) h_{\omega}(\varepsilon)^{-1} \\
& =w_{\beta}\left(\varepsilon^{(\beta, \omega)} \eta\right) w_{\beta}\left(-\varepsilon^{(\beta, \omega)}\right)=h_{\beta}(\eta) .
\end{aligned}
$$

We have not stipulated that $h_{\omega}(\varepsilon)$ is semisimple. However, as we shall see in the next section, this automatically follows from $(1)$, whenever $h_{\omega}(\varepsilon)$ is identified with its image in the group GL $(V)$ for some finite-dimensional irreducible representation $V$ of the Chevalley group $G$. Since $h_{\omega}(\varepsilon)$ is only defined up to a central factor, we may assume from the very start that $h_{\omega}(\varepsilon)$ is semisimple.

In the adjoint case, (1) already determines the element $h_{\omega}(\varepsilon)$ uniquely, because here $h_{\omega}(\varepsilon)=h_{\mathrm{ad}}\left(\chi_{\omega, \varepsilon}\right)$. As has been mentioned, in the simply connected case the element $h_{\omega}(\varepsilon)$ is defined by this formula only up to a central factor. Let $C$ denote the center of the extended Chevalley group $\bar{G}=\bar{G}(\Phi, K)$. In most calculations, the element $h_{\omega}(\varepsilon)$ can be viewed as a coset modulo $C$, rather than a genuine element of the group $\bar{G}$. However, since a central factor does not affect the element $w$ in the Bruhat decomposition, we tend to speak of $h_{\omega}(\varepsilon)$ as genuine elements. Another justification of such a viewpoint is that the construction in [40] makes it possible to specify central factors so that for classical cases the resulting elements coincide with the usual elements described in the next section.

As a matter of fact, we wish $h_{\omega}(\varepsilon)$ to be linear in $\omega$, i.e., to satisfy the following two identities:

$$
h_{\omega_{1}+\omega_{2}}(\varepsilon)=h_{\omega_{1}}(\varepsilon) h_{\omega_{2}}(\varepsilon), \quad h_{-\omega}(\varepsilon)=h_{\omega}(\varepsilon)^{-1} .
$$

Thus, we only need to specify $h_{\omega}(\varepsilon)$ for a generating system of $P\left(\Phi^{\vee}\right)$, say, for the fundamental weights $\omega=\varpi_{r}\left(\Phi^{\vee}\right)$.

On top of that, we wish $h_{\omega}(\varepsilon)$ to be compatible with the action of the Weyl group, i.e., to satisfy the identity

$$
h_{w \omega}(\varepsilon)=n_{w} h_{\omega}(\varepsilon) n_{w}^{-1} .
$$

Thus, all $h_{\omega}(\varepsilon)$ 's are uniquely determined by one of them, namely, by that corresponding to a weight $\omega$ whose Weyl orbit generates the entire weight lattice $P\left(\Phi^{\vee}\right)$. In the next section we illustrate this choice in classical examples.

\section{$\S 5$. Classical cases}

In this section we describe what weight elements look like in the natural representations of classical groups.

- Let $\Phi=\mathrm{A}_{l}$. Then $\Phi^{\vee}=\Phi$. Consider the group $G=G(\Phi, K)$ in the usual vector representation of dimension $l+1$, i.e., the representation with the highest weight $\varpi_{1}$. This representation furnishes the simply connected group $\mathrm{SL}(n, K)$. For a fundamental weight $\omega=\varpi_{k}\left(\mathrm{~A}_{l}\right)$, set

$$
h_{\omega}(\varepsilon)=\operatorname{diag}(\varepsilon, \ldots, \varepsilon, 1, \ldots, 1),
$$

where the number of $\varepsilon$ 's equals $k$. It is obvious that these elements act on $x_{\beta}(\xi)$ by formula (1). Together with linearity, this choice determines $h_{\omega}(\varepsilon)$ uniquely for any weight $\omega$. Additionally imposing compatibility with the action of the Weyl group, we only need to specify $h_{\varpi_{1}}(\varepsilon)$. Since $(l+1) \varpi_{1} \in Q\left(\mathrm{~A}_{l}\right)$, the element $h_{(l+1) \varpi_{1}}(\varepsilon)=h_{\varpi_{1}}\left(\varepsilon^{l+1}\right)$ should lie in $H$, up to a central factor. This is indeed the case. Recall that in this representation the semisimple root elements are conjugate to

$$
h_{e_{1}-e_{2}}(\varepsilon)=\operatorname{diag}\left(\varepsilon, \varepsilon^{-1}, 1, \ldots, 1\right) .
$$

Clearly,

$$
h_{\varpi_{1}}\left(\varepsilon^{l+1}\right)=\varepsilon h_{e_{1}-e_{2}}(\varepsilon) \cdots h_{e_{1}-e_{l}}(\varepsilon) .
$$


The group generated by $\operatorname{SL}(n, K)$ and all $h_{\varpi_{1}}(\varepsilon), \varepsilon \in K^{*}$, is the general linear group GL $(n, K)$, which may thus be viewed as the extended Chevalley group of type $\mathrm{A}_{l}$. The weight elements $h_{\varpi_{1}}(\varepsilon)=d_{1}(\varepsilon)$ and their conjugates are the usual pseudoreflections.

- Let $\Phi=\mathrm{B}_{l}$. Then $\Phi^{\vee}=\mathrm{C}_{l}$. Again, consider the group $G=G(\Phi, K)$ in the usual $(2 l+1)$-dimensional representation, i.e., the first fundamental one. This representation furnishes the group $\mathrm{SO}(2 l+1, K)$, the adjoint Chevalley group of type $\mathrm{B}_{l}$. For $\omega=$ $\varpi_{k}\left(\mathrm{C}_{l}\right)$, set

$$
h_{\omega}(\varepsilon)=\operatorname{diag}\left(\varepsilon, \ldots, \varepsilon, 1, \ldots, 1, \varepsilon^{-1}, \ldots, \varepsilon^{-1}\right),
$$

where both the number of $\varepsilon$ 's and the number of $\varepsilon^{-1}$ 's are equal to $k$. This identifies $h_{\omega}(\varepsilon)$ almost uniquely for any weight $\omega$. In fact, this representation is not faithful, so strictly speaking we should describe elements $h_{\omega}(\varepsilon)$ in the spin representation. As before, the most interesting weight elements are $h_{\varpi_{1}}(\varepsilon)$. If we require compatibility with the action of the Weyl group, then the choice of $h_{\varpi_{1}}(\varepsilon)$ uniquely determines the images of all other weight elements in this representation. Since $\varpi_{1}$ generates $P\left(\mathrm{C}_{l}\right)$ modulo $Q\left(\mathrm{C}_{l}\right)$, these elements generate $T$ modulo $H$. In fact, $H$ is generated by semisimple long root elements, which in this case are conjugate to $h_{\varpi_{2}}(\varepsilon)=h_{e_{1}+e_{2}}(\varepsilon)$. Since $2 \varpi_{1} \in Q\left(\mathrm{C}_{l}\right)$, the elements $h_{2 \varpi_{1}}(\varepsilon)=h_{\varpi_{1}}\left(\varepsilon^{2}\right)$ should, modulo a central factor, lie in $H$ for any $\varepsilon \in K^{*}$. In this case they lie in $H$ itself. Indeed,

$$
h_{\varpi_{1}}\left(\varepsilon^{2}\right)=h_{e_{1}+e_{2}}(\varepsilon) h_{e_{1}-e_{2}}(\varepsilon)
$$

where

$$
h_{e_{1}-e_{2}}(\varepsilon)=\operatorname{diag}\left(\varepsilon, \varepsilon^{-1}, 1, \ldots, 1, \varepsilon, \varepsilon^{-1}\right) .
$$

The elements $h_{\varpi_{1}}(\varepsilon), \varepsilon \in K^{*}$, already belong to the Chevalley group $\mathrm{SO}(2 l+1, K)$ in this case, which shows that it is already extended in the above sense. The only possible further diagonal extension by scalar matrices, the general orthogonal group $\mathrm{GO}(2 l+1, K)$, is trivial. But the spin group $\operatorname{Spin}(2 l+1, K)$ admits a nontrivial diagonal extension that covers $\mathrm{SO}(2 l+1, K)$. At the same time, $\operatorname{Spin}(2 l+1, K)$ itself only covers $\Omega(2 l+1, K)$. In [40, one can find a construction of this extension and its identification in terms of the Clifford group.

- Let $\Phi=\mathrm{C}_{l}$. Then $\Phi^{\vee}=\mathrm{B}_{l}$. The usual $2 l$-dimensional representation, the first fundamental one, furnishes the simply connected Chevalley group $\operatorname{Sp}(2 l, K)$. For $\omega=$ $\varpi\left(\mathrm{B}_{l}\right), k \leq l-1$, the corresponding weight elements $h_{\omega}(\varepsilon)$ have the same form as those described above for $\Phi=\mathrm{B}_{l}$, while for $\omega=\varpi_{l}\left(\mathrm{~B}_{l}\right)$ we may choose

$$
h_{\omega}(\varepsilon)=\operatorname{diag}(\varepsilon, \ldots, \varepsilon, 1, \ldots, 1),
$$

where both the number of $\varepsilon$ 's and the number of 1's are equal to $l$. Since in this case $h_{\varpi_{1}}(\varepsilon)$ is a semisimple long root element, all $h_{\varpi_{k}}(\varepsilon), 1 \leq k \leq l-1$, belong to $H$. This is not true for $h_{\varpi_{l}}(\varepsilon)$, however. Namely, since $2 \varpi_{l} \in Q\left(\mathrm{~B}_{l}\right)$, the square of $h_{\varpi_{l}}(\varepsilon)$ is in $H$ modulo the center. Indeed,

$$
h_{\varpi_{l}}\left(\varepsilon^{2}\right)=\varepsilon \operatorname{diag}\left(\varepsilon, \ldots, \varepsilon, \varepsilon^{-1}, \ldots, \varepsilon^{-1}\right),
$$

where both the number of $\varepsilon$ 's and the number of $\varepsilon^{-1}$ 's are equal to $l$. The second factor is the product of semisimple root elements $h_{\alpha}(\varepsilon)$, taken over all positive long roots. Now, the group generated by $\operatorname{Sp}(2 l, K)$ and all $h_{\varpi_{l}}(\varepsilon), \varepsilon \in K^{*}$, is the general symplectic group $\operatorname{GSp}(2 l, K)$, which thus may be viewed as the extended Chevalley group of type $\mathrm{C}_{l}$.

- Let $\Phi=\mathrm{D}_{l}$. Then $\Phi^{\vee}=\Phi$. The usual $2 l$-dimensional representation, the first fundamental one, furnishes the group $\mathrm{SO}(2 l, K)$. For $\omega=\varpi\left(\mathrm{D}_{l}\right), k \leq l-2$, the corresponding weight elements $h_{\omega}(\varepsilon)$ have the same form as above for $\Phi=\mathrm{B}_{l}$, and for the role of $\omega=\varpi_{l}\left(D_{l}\right)$ we may choose the same element as for $\varpi_{l}\left(C_{l}\right)$. Finally, for $\omega=\varpi_{l-1}\left(D_{l}\right)$ we set

$$
h_{\omega}(\varepsilon)=\operatorname{diag}(\varepsilon, \ldots, \varepsilon, 1, \varepsilon, 1, \ldots, 1),
$$


where both the number of $\varepsilon$ 's and the number of 1's are equal to $l$. Essentially, this case is very close to the cases of $\mathrm{B}_{l}$ and $\mathrm{C}_{l}$ already considered. There are some small distinctions, though. Namely, if $l$ is odd, then $4 \varpi_{k} \in Q\left(\mathrm{D}_{l}\right), k=l-1, l$, but $2 \varpi_{k} \notin Q\left(\mathrm{D}_{l}\right)$. In this case we do not have $h_{\varpi_{1}}(\varepsilon)$ at our disposal, but only the semisimple root elements $h_{\varpi_{2}}(\varepsilon)$. Thus, it is impossible to present $h_{\omega}\left(\varepsilon^{2}\right)$ as a product of an element of $H$ and a central factor. On the other hand, since $h_{\varpi_{1}}\left(\varepsilon^{2}\right)$ always belongs to $H$, we can, in the same way as in the $\mathrm{C}_{l}$ case, express $h_{\omega}\left(\varepsilon^{4}\right)$ as a product of an element of $H$ and a central factor. As opposed to that, if $l$ is even, then already $h_{\omega}\left(\varepsilon^{2}\right)$ itself can be expressed as the product $\varepsilon h_{e_{1}+e_{2}}(\varepsilon) h_{e_{3}+e_{4}}(\varepsilon) \cdots h_{e_{l-1}+e_{l}}(\varepsilon)$. The special orthogonal group $\mathrm{SO}(2 l, K)$ is already extended by some, but in general not all, diagonal automorphisms. Here, as in the $\mathrm{B}_{l}$ case, the image of the simply connected group coincides with the kernel of the spinorial norm $\Omega(2 l, K)$. A further diagonal extension is furnished by the product of $\mathrm{SO}(2 l, K)$ and all $h_{\varpi_{l}}(\varepsilon), \varepsilon \in K^{*}$, which is the general orthogonal group $\operatorname{GO}(2 l, K)$. This last group may thus be viewed as an extended Chevalley group of type $\mathrm{D}_{l}$. In [40] one can find a construction of a similar diagonal extension of the spin group $\operatorname{Spin}(2 l, K)$ and its identification in terms of the Clifford group.

\section{§6. Berman-Moody COnstruction}

In this section we recall a construction of the extended maximal torus $\bar{T}_{\mathrm{sc}}$ in the simply connected case, under the assumption that the quotient group $P(\Phi) / Q(\Phi) \cong \mathbb{Z} / m \mathbb{Z}$ is cyclic.

Fix a weight $\lambda$ generating $P(\Phi)$ modulo $Q(\Phi)$. Then $P(\Phi)=\mathbb{Z} \lambda+Q(\Phi)$. Embed the l-dimensional lattice $Q(\Phi)=\mathbb{Z} \alpha_{1} \oplus \cdots \oplus \mathbb{Z} \alpha_{l}$ into the $(l+1)$-dimensional lattice

$$
\bar{P}=\mathbb{Z} \bar{\alpha} \oplus \mathbb{Z} \alpha_{1} \oplus \cdots \oplus \mathbb{Z} \alpha_{l} .
$$

Then the group $\bar{T}_{\mathrm{sc}}=\bar{T}_{\mathrm{sc}}(\Phi, K)$ is defined as $\bar{T}_{\mathrm{sc}} \cong \operatorname{Hom}\left(\bar{P}, K^{*}\right)$.

Although these groups are isomorphic, sometimes it is convenient to distinguish elements of $\bar{T}_{\mathrm{sc}}$ from those of $\operatorname{Hom}\left(\bar{P}, K^{*}\right)$. We do this in the same way as for $T_{\mathrm{ad}}$. Namely, we write $h(\chi)=h_{\mathrm{sc}}(\chi)$ for the element of $\bar{T}_{\mathrm{sc}}$ corresponding to a $K$-character $\chi \in \operatorname{Hom}\left(\bar{P}, K^{*}\right)$. For a character $\chi$ we denote by $\chi^{\text {res }}$ its restriction to $Q(\Phi)$. Now, we can define a homomorphism res : $\bar{T}_{\mathrm{sc}} \rightarrow \bar{T}_{\mathrm{ad}}$ sending an element $h_{\mathrm{sc}}(\chi) \in \bar{T}_{\mathrm{sc}}$ to $h_{\text {ad }}\left(\chi^{\text {res }}\right) \in \bar{T}_{\text {ad }}$. Clearly, res is in fact an epimorphism, while its kernel coincides with the set of all $h(\chi)$ such that $\chi\left(\alpha_{i}\right)=1$ for all $1 \leq i \leq l$. For any $\varepsilon \in K^{*}$, we define a character $\chi_{\varepsilon} \in \operatorname{Hom}\left(\bar{P}, K^{*}\right)$ by

$$
\chi_{\varepsilon}(\bar{\alpha})=\varepsilon^{-1} ; \quad \chi_{\varepsilon}\left(\alpha_{i}\right)=1, \quad 1 \leq i \leq l .
$$

Then the assignment $\varepsilon \mapsto \chi_{\varepsilon}$ gives rise to a homomorphism $\delta: K^{*} \rightarrow \bar{T}_{\mathrm{sc}}$ forming, together with res, a short exact sequence. Now, we define an embedding incl of the group $\operatorname{Hom}\left(P(\Phi), K^{*}\right)$ into the group $\operatorname{Hom}\left(\bar{P}, K^{*}\right)$, by setting

$$
\operatorname{incl}(\chi)(\bar{\alpha})=\chi(\lambda)^{-1} ; \quad \operatorname{incl}(\chi)\left(\alpha_{i}\right)=\chi\left(\alpha_{i}\right), \quad 1 \leq i \leq l,
$$

for a $\chi \in \operatorname{Hom}\left(P(\Phi), K^{*}\right)$. By definition, incl depends on $\lambda$, but in [40] it was shown that, apart from the case of $\mathrm{A}_{l}$, the resulting groups $\bar{G}_{\mathrm{sc}}$ do not depend on $\lambda$. Now, let $m \lambda \in Q(\Phi)$, i.e., $m \lambda=m_{1} \alpha_{1}+\cdots+m_{l} \alpha_{l}$, where $m_{i} \in \mathbb{Z}$ and the greatest common divisor of $m, m_{1}, \ldots, m_{l}$ equals 1 . This allows us to define a homomorphism det $: \bar{T}_{\mathrm{sc}} \rightarrow K^{*}$ sending an element $h(\chi)$ to

$$
\operatorname{det}(h(\chi))=\chi\left(-m \bar{\alpha}-m_{1} \alpha_{1}-\cdots-m_{l} \alpha_{l}\right) .
$$

The homomorphisms incl and det form a short exact sequence; together with the above homomorphisms and the obvious homomorphisms they can be included in the following 
commutative diagram:

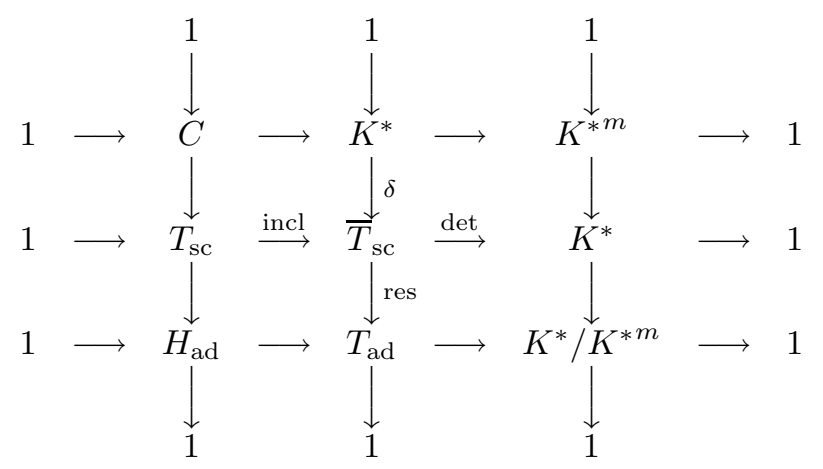

Next, we define an action of $\bar{T}_{\mathrm{sc}}$ on the Chevalley group $G_{\mathrm{sc}}=G_{\mathrm{sc}}(\Phi, K)$. Namely, we make $h(\chi), \chi \in \operatorname{Hom}\left(\bar{P}, K^{*}\right)$, act on $G_{\mathrm{sc}}$ via the diagonal automorphism $\phi_{\chi^{\text {res }}}$; see $\S 3$. Thus, we get a homomorphism $\psi$ of the group $\bar{T}_{\text {sc }}$ into the automorphism group $\operatorname{Aut}\left(G_{\mathrm{sc}}\right)$ of the group $G_{\mathrm{sc}}$, which can be included in the following commutative square:

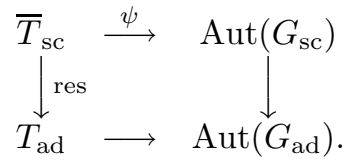

Now we are all set to construct the extended simply connected Chevalley group $\bar{G}_{\mathrm{sc}}$. Namely, we put $\bar{G}_{\mathrm{sc}}=\left(G_{\mathrm{sc}} \lambda \bar{T}_{\mathrm{sc}}\right) / M$, where $M$ is a central normal subgroup of the semidirect product $G_{\mathrm{sc}} \lambda \bar{T}_{\mathrm{sc}}$ defined by

$$
M=\left\{(g, h) \mid g \in T_{\mathrm{sc}} \leq G_{\mathrm{sc}}, h \in \bar{T}_{\mathrm{sc}}, \operatorname{incl}(g)=h^{-1}\right\} .
$$

It is easy to check (this was done in [40]) that for $\bar{G}_{\text {sc }}$ we get the same commutative diagram with exact columns and rows as for $\bar{T}_{\mathrm{sc}}$. One only has to replace $T$ by $G$ and $H$ by $E$ in the above diagram. It is easy to adapt this construction to the case where the quotient group $P(\Phi) / Q(\Phi)$ is not cyclic; one only has to start with a lattice $\bar{Q}$ of rank $l+2$.

Now we can eliminate all ambiguity in the definition of $h_{\omega}(\varepsilon), \omega \in P\left(\Phi^{\vee}\right)$, by setting $h_{\omega}(\varepsilon)=h_{\mathrm{sc}}(\chi)$ for a character $\chi \in \operatorname{Hom}\left(\bar{P}, K^{*}\right)$ such that $\chi\left(\alpha_{i}\right)=\varepsilon^{\left(\alpha_{i}, \omega\right)}, 1 \leq i \leq l$, or, in other words, $\chi^{\text {res }}=\chi_{\omega, \alpha}$. To uniquely specify the character $\chi$, we need to define the value of $\chi(\bar{\alpha})$. We may set, for instance, $\chi(\bar{\alpha})=1$ or $\chi(\bar{\alpha})=\chi\left(\alpha_{1}\right)^{-1} \cdots \chi\left(\alpha_{l}\right)^{-1}$, etc. The correct choice of $\chi(\bar{\alpha})$ identifies our det either with the usual determinant, or with the spinorial norm, or with something similar.

Observe that the resulting groups $\bar{G}_{\text {sc }}$ are usually well known. For classical cases they can be interpreted either as the similarity groups of the corresponding bilinear forms or in terms of the corresponding Clifford groups. There are similar interpretations also for the types $\mathrm{E}_{6}$ and $\mathrm{E}_{7}$. Namely, it is known that the simply connected groups $G\left(\mathrm{E}_{6}, K\right)$ and $G\left(\mathrm{E}_{7}, K\right)$ are isomorphic to the isometry groups of a certain 3-linear form on a 27-dimensional space and, respectively, of a pair consisting of a symplectic form and a certain 4-linear form on a 56-dimensional space. Such constructions go back to the works of Dickson, Chevalley, Freudenthal, Springer and others and were finalized by Aschbacher [38] (see [76, where many further references can be found). In these cases the corresponding extended groups $\bar{G}\left(\mathrm{E}_{6}, K\right)$ and $\bar{G}\left(\mathrm{E}_{7}, K\right)$ may be interpreted as the groups of transformations preserving the above forms up to similarity. 


\section{$\S 7$. WeIGHT ELEMENTS IN IRREDUCIBLE REPRESENTATIONS}

In this section we describe the action of weight elements in irreducible representations.

Let $\pi$ be an irreducible representation of the complex semisimple Lie algebra $L$ of type $\Phi$, and let $V_{K}$ be the corresponding Weyl module of the Chevalley group $G=G(\Phi, K)$. Here we present another construction of weight elements, by specifying their action on $V_{K}$.

Theorem 2. A weight element $h_{\omega}(\varepsilon), \omega \in P\left(\Phi^{\vee}\right), \varepsilon \in K^{*}$, acts on every weight subspace $V^{\lambda}, \lambda \in \Lambda(\pi)$, as the scalar multiplication by $c_{\lambda}=c_{\lambda}(\omega, \varepsilon) \neq 0$. For any two weights $\lambda, \mu \in \Lambda(\pi)$, we have

$$
c_{\lambda} c_{\mu}^{-1}=\varepsilon^{(\lambda, \omega)-(\mu, \omega)} .
$$

Proof. We prove this theorem by induction on $\lambda$ with respect to the usual order on $\Lambda(\pi)$. Recall that we write $\lambda>\mu$ if and only if $\lambda-\mu=m_{1} \alpha_{1}+\cdots+m_{l} \alpha_{l}$ is a linear combination of fundamental roots with nonnegative integral coefficients. Among the weights of $\pi$ there is a unique weight maximal with respect to this order, called the highest weight of this representation and denoted by $\lambda_{0}$. It suffices to prove (2) only for $\mu=\lambda_{0}$. First, observe that $h_{\omega}(\varepsilon)$ commutes with $H$ elementwise, and thus it stabilizes all weight subspaces $V^{\lambda}$, $\lambda \in \Lambda(\pi)$. In the case where $\lambda=\lambda_{0}$ there is nothing to prove. Indeed, the corresponding weight space $V^{\lambda_{0}}$ is one-dimensional and (2) is vacuous. Assume that for all $\mu>\lambda$ we have already proved that $V^{\mu}$ is generated by eigenvectors of $h_{\omega}(\varepsilon)$ corresponding to the eigenvalues $c_{\mu}$. Moreover, assume that formula (2) is true for all pairs $(\mu, \nu), \mu, \nu>\lambda$, or, what is the same, for all pairs $\left(\mu, \lambda_{0}\right), \mu>\lambda$. We wish to prove that then the same is true also for $\lambda$.

First, observe that $x_{\alpha}(\xi)=\exp \left(\xi e_{\alpha}\right)$ with nilpotent $e_{\alpha}$, and thus formula (1) implies that

$$
h_{\omega}(\varepsilon) e_{\alpha} h_{\omega}(\varepsilon)^{-1}=\varepsilon^{(\alpha, \omega)} e_{\alpha}
$$

Now, since the representation $\pi$ is irreducible, the weight subspace $V^{\lambda}$ is spanned by the vectors $e_{-\alpha} v$, where $v \in V^{\lambda+\alpha}, \alpha \in \Phi^{+}, \lambda+\alpha \in \Lambda(\pi)$. By the inductive hypothesis, all $v \in V^{\lambda+\alpha}$ are eigenvectors of $V$ with the same eigenvalue $c_{\lambda+\alpha}$. Now, (3) implies that $e_{-\alpha} v$ is an eigenvector of $h_{\omega}(\varepsilon)$ with the eigenvalue $\varepsilon^{-(\alpha, \omega)} c_{\lambda+\alpha}$. This means that $V^{\lambda}$ is spanned by eigenvectors of $h_{\omega}(\varepsilon)$, so that the restriction of $h_{\omega}(\varepsilon)$ to this subspace is semisimple. It only remains to observe that the corresponding eigenvalues coincide, i.e., $\varepsilon^{-(\alpha, \omega)} c_{\lambda+\alpha}=\varepsilon^{-(\beta, \omega)} c_{\lambda+\beta}$ for all $\alpha, \beta \in \Phi^{+}$such that $\lambda+\alpha, \lambda+\beta \in \Lambda(\pi)$. But this is precisely the inductive hypothesis. Finally, set $c_{\lambda}=\varepsilon^{-(\alpha, \omega)} c_{\lambda+\alpha}$ for some $\alpha \in \Phi^{+}$. As we have proved, the value $c_{\lambda}$ does not depend on the choice of $\alpha \in \Phi^{+}$such that $\lambda+\alpha \in \Lambda(\pi)$. Then, clearly,

$$
c_{\lambda} c_{\lambda_{0}}^{-1}=\varepsilon^{-(\alpha, \omega)} c_{\lambda+\alpha} c_{\lambda_{0}}^{-1}=\varepsilon^{-(\alpha, \omega)+(\lambda+\alpha, \omega)-\left(\lambda_{0}, \omega\right)}=\varepsilon^{(\lambda, \omega)-\left(\lambda_{0}, \omega\right)},
$$

and this finishes the proof of the theorem.

If we require additionally that the eigenvalues of $h_{\omega}(\varepsilon)$ be powers of $\varepsilon$, then $h_{\omega}(\varepsilon)$ is determined by this theorem up to a scalar factor $\varepsilon^{m}$. Now, the requirement that $h_{\omega}(\varepsilon)$ have the largest possible multiplicity of 1 as an eigenvalue, or that $h_{\omega}(\varepsilon)$ be linear with respect to $\omega$, suffices to determine it uniquely.

For example, in the usual 27-dimensional representation of the Chevalley group $G=$ $G\left(\mathrm{E}_{6}, K\right)$, i.e., the representation with the highest weight $\varpi_{1}$, an appropriate weight element $h_{\varpi_{1}}(\varepsilon)$ has one eigenvalue $\varepsilon, 16$ eigenvalues 1 , and 10 eigenvalues $\varepsilon^{-1}$ (see 76 , $\S 6]$, where this example is considered in some detail).

Of course, there is no way to prove a similar result for reducible representations. First, the difference of two weights of a reducible representation does not necessarily belong 
to the root lattice $Q(\Phi)$, so that the exponent of $\varepsilon$ on the right-hand side is not an integer. Indeed, there is no a priori relationship among the eigenvalues of $h_{\omega}(\varepsilon)$ on various irreducible components. Even worse than that, it is not at all obvious why $h_{\omega}(\varepsilon)$ should be semisimple. In fact, the semisimplicity of $h_{\omega}(\varepsilon)$ in a reducible representation does not follow from (1); it should be imposed separately.

\section{$\S 8$. Generation of the maximal torus $\bar{T}_{\mathrm{sc}}$}

In this section we study the generation of $\bar{T}_{\mathrm{sc}}$ by the Weyl orbit of a single weight, which allows us to specify all $h_{\omega}(\varepsilon)$ once we know one of them. It turns out that this happens rather often, at least modulo the center. In fact, using the explicit construction described in previous sections, we can normalize these elements is such a way that they generate the extended torus.

Lemma 1. Let $\omega_{1}, \ldots, \omega_{s}$ be a set of weights generating $P\left(\Phi^{\vee}\right)$. Then the corresponding weight elements $h_{\omega_{1}}(\varepsilon), \ldots, h_{\omega_{s}}(\varepsilon)$ generate the group $\bar{T}$ modulo the center of the group $\bar{G}$.

Proof. This immediately follows from the definitions. If $\omega=m_{1} \omega_{1}+\cdots+m_{s} \omega_{s}$, then the conjugation action of

$$
h=h_{\omega_{1}}(\varepsilon)^{m_{1}} \cdots h_{\omega_{s}}(\varepsilon)^{m_{s}}
$$

on the root unipotents $x_{\alpha}(\xi)$ coincides with the conjugation action of $h_{\omega}(\varepsilon)$ on these elements. Thus, $h$ and $h_{\omega}(\varepsilon)$ coincide up to a central factor.

Recall that $\Phi_{l}$ and $\Phi_{s}$ denote the sets of long and short roots in $\Phi$, respectively.

Lemma 2. Suppose that a weight $\omega$ of the root system $\Phi^{\vee}$ possesses the following two properties:

- $\omega$ generates $P\left(\Phi^{\vee}\right)$ over $Q\left(\Phi^{\vee}\right)$, and

- the greatest common divisor $\operatorname{gcd}(\omega, \alpha)$ of the inner products $(\omega, \alpha), \alpha \in \Phi_{l}$, coincides with 1.

Then the lattice $P$ generated by the Weyl orbit $W\left(\Phi^{\vee}\right) \omega$ of the weight $\omega$ coincides with $P\left(\Phi^{\vee}\right)$.

Proof. We only need to prove that $P \geq Q\left(\Phi^{\vee}\right)$. Then our claim follows, in view of the first condition. Indeed, for any root $\alpha^{\vee} \in \Phi^{\vee}$ we have $w_{\alpha^{\vee}}(\omega)-\omega=(\omega, \alpha) \alpha^{\vee} \in P$. Clearly, the lattice $P(\Phi)$ contains all vectors $(\omega, \alpha) \alpha^{\vee}=\omega-w_{\alpha} \omega$. Since $P$ is invariant under the action of the Weyl group $W=W\left(\Phi^{\vee}\right)$ and the greatest common divisor of $(\omega, \alpha), \alpha \in \Phi_{l}$, equals $1, P$ contains all dual roots $\alpha^{\vee}, \alpha \in \Phi_{l}$. Obviously, these roots generate $Q\left(\Phi^{\vee}\right)$.

These two lemmas immediately imply the following result.

Proposition 1. Suppose a weight $\omega$ of the root system $\Phi^{\vee}$ satisfies the conditions of Lemma 2. Then

$$
\bar{T}_{\mathrm{sc}}(\Phi, K)=C\left\langle h_{\omega}(\varepsilon), \omega \in W(\Phi) \varpi_{i}, \varepsilon \in K^{*}\right\rangle,
$$

where $C$ is the center of the group $\bar{G}(\Phi, K)$.

Again, especially significant is the case where $\omega=\varpi_{i}$ is an appropriate fundamental weight. In particular, Proposition 1 implies that $\bar{T}_{\mathrm{sc}}$ is generated by the Weyl orbit of $h_{\varpi_{1}}(\varepsilon)$ for $\Phi=\mathrm{A}_{l}, \mathrm{~B}_{l}, \mathrm{E}_{6}$, by the orbit of $h_{\varpi_{7}}(\varepsilon)$ for $\Phi=\mathrm{E}_{7}$, and by the orbit of $h_{\varpi_{l}}(\varepsilon)$ for $\Phi=\mathrm{C}_{l}$. For $\Phi=\mathrm{D}_{l}$, generally speaking, none of the Weyl orbits of $h_{\varpi_{1}}(\varepsilon)$, $h_{\varpi_{l-1}}(\varepsilon)$, or $h_{\varpi_{l}}(\varepsilon)$ alone spans the entire extended torus, but any two of them do. This explains why Theorem 1 of the present paper naturally arises in the study of subgroups containing $\bar{T}$. 
Take a one-parameter subgroup of weight elements whose Weyl orbit generates the whole torus and consider its conjugates. The symplectic case is an exception, because the elements $h_{\varpi_{l}}(\varepsilon)$ are fairly complicated. In fact, in all classical cases we work with $h_{\varpi_{1}}(\varepsilon)$. These are microweight elements for $\mathrm{A}_{l}, \mathrm{~B}_{l}, \mathrm{D}_{l}$ and semisimple long root elements for $\mathrm{C}_{l}$. For $\mathrm{E}_{6}$ we work with $h_{\varpi_{1}}(\varepsilon)$, or, what is essentially the same, with $h_{\varpi_{6}}(\varepsilon)$. Finally, for $\mathrm{E}_{7}$ we work with $h_{\varpi_{7}}(\varepsilon)$.

\section{§. INDUCED DIAGONAL AUTOMORPHISMS}

In this section we calculate which diagonal automorphisms are induced by a Chevalley group acting by conjugation on a regularly embedded Chevalley subgroup. This gives us one more construction of the extended Chevalley group $\bar{G}_{\mathrm{sc}}$ of types $\mathrm{E}_{6}$ and $\mathrm{E}_{7}$.

First, let $\Phi$ be any root system, and let $\Delta$ be its subsystem. Then some Chevalley group $G_{\Delta}=G(\Delta, K)$ of type $\Delta$ naturally embeds in $G_{\Phi}=G(\Phi, K)$. This is what Dynkin calls a regular embedding, roots to roots. Notice though that even for an adjoint group $G_{\Phi}$ its regularly embedded subgroup of type $\Delta$ may be simply connected. A natural question is which diagonal automorphisms of $G_{\Delta}$ are induced by the inner diagonal automorphisms of $G_{\Phi}$. The answer to this question is given by the following result, which will be used in the proof of Proposition 3.

Theorem 3. Let $\Phi$ be an irreducible root system, and let $\Delta$ be its irreducible subsystem. Almost always conjugation by elements of the group $T_{\mathrm{sc}}(\Phi, K)$ induces all diagonal

automorphisms on the regularly embedded subgroup $G(\Delta, K) \leq G_{\mathrm{sc}}(\Phi, K)$. All possible exceptions are listed in Table 2.

\section{TABLE 2}

$\begin{array}{lll}\Phi & \Delta & h_{\omega}(\varepsilon) \\ \mathrm{B}_{l}: & \mathrm{A}_{l-1}, l \text { even } & h_{\varpi_{2}}(\varepsilon) \\ & \mathrm{D}_{l} & - \\ & \mathrm{D}_{k}, 3 \leq k \leq l-1 & h_{\varpi_{1}}(\varepsilon) \\ \mathrm{C}_{l}: & \mathrm{C}_{k}, 1 \leq k \leq l-1 & - \\ \mathrm{D}_{l}: & \mathrm{A}_{l-1}^{\prime} \text { and } \mathrm{A}_{l-1}^{\prime \prime}, l \text { even } & h_{\varpi_{2}}(\varepsilon) \\ & \mathrm{D}_{k}, 3 \leq k \leq l-1 & h_{\varpi_{1}}(\varepsilon) \\ \mathrm{E}_{6}: & \mathrm{A}_{5} & h_{\varpi_{3}}(\varepsilon) \\ \mathrm{E}_{7}: & \mathrm{A}_{7} & h_{\varpi_{4}}(\varepsilon) \\ & \mathrm{A}_{5}^{\prime \prime} & h_{\varpi_{2}}(\varepsilon) \\ & \mathrm{D}_{6} & h_{\varpi_{6}}(\varepsilon) \\ \mathrm{E}_{8}: & \mathrm{A}_{8} & h_{\varpi_{3}}(\varepsilon) \\ & \mathrm{D}_{8} & h_{\varpi_{8}}(\varepsilon) \\ & \mathrm{A}_{7}^{\prime \prime} & h_{\varpi_{2}}(\varepsilon) \\ \mathrm{F}_{4}: & \mathrm{B}_{4} & - \\ & \mathrm{D}_{4} & - \\ & \mathrm{A}_{3} & h_{\varpi_{2}}(\varepsilon) \\ \mathrm{G}_{2}: & \mathrm{A}_{2} & -\end{array}$

The last column of this table lists the diagonal automorphisms of $G(\Delta, K)$ that are induced by internal diagonal automorphisms of the group $G(\Phi, K)$. In particular, '-' in this column means that $\bar{T}_{\text {sc }}$ does not induce any diagonal automorphism on $G(\Delta, K)$ apart from the internal ones. 
Proof. To determine which diagonal automorphisms of $G(\Delta, K)$ are induced by the inner diagonal automorphisms of $G_{\mathrm{sc}}(\Phi, K)$, we recall that the group $T_{\mathrm{sc}}$ is generated by the semisimple root elements $h_{\alpha}(\varepsilon), \alpha \in \Pi$. Thus, we only need to determine which diagonal automorphisms are induced on $G(\Delta, K)$ by $h_{\alpha}(\varepsilon), \alpha \in \Pi, \alpha \notin \Delta$. We do this case by case.

- When $\Phi=\mathrm{A}_{l}$, it is clear that $h_{\varpi_{k+1}}\left(\varepsilon^{-1}\right)$ induces on $\Delta=\left\langle\alpha_{1}, \ldots, \alpha_{k}\right\rangle$ the diagonal automorphism corresponding to $h_{\varpi_{k}}(\varepsilon)$. This automorphism generates $\bar{T}_{\text {sc }}$ modulo the inner ones.

- The system $\Phi=\mathrm{B}_{l}$ has the following proper irreducible subsystems: $\mathrm{A}_{k}, 1 \leq k \leq$ $l-1 ; \mathrm{B}_{k}, 1 \leq k \leq l-1 ; \mathrm{D}_{k}, 1 \leq k \leq l$. In the case of $\Delta=\mathrm{A}_{k}, 1 \leq k \leq l-2$, all diagonal automorphisms are already induced in $\mathrm{A}_{l-1}$. For $\Delta=\mathrm{A}_{l-1}$, the short fundamental root induces on $G(\Delta, K)$ the diagonal automorphism corresponding to $h_{\varpi_{l-1}}\left(\varepsilon^{2}\right)$ - in fact this automorphism is induced already in $\mathrm{D}_{l}$. But this automorphism generates the same group as $h_{\varpi_{l-2}}(\varepsilon)$ modulo the inner automorphisms of $G\left(\mathrm{~A}_{l-1}, K\right)$. Indeed,

$$
\left(\begin{array}{ll}
\varepsilon^{2} & \\
& 1
\end{array}\right)\left(\begin{array}{ll}
\varepsilon^{-1} & \\
& \varepsilon
\end{array}\right)=\left(\begin{array}{ll}
\varepsilon & \\
& \varepsilon
\end{array}\right) \text {. }
$$

Thus, the answer depends on the parity of $l$. If $l$ is odd, this group coincides with the entire group of diagonal automorphisms of $G_{\Delta}$, but if $l$ is even, it has index 2 in that group. Clearly, the root $\alpha_{l-k}$ is orthogonal to all fundamental roots of $\mathrm{B}_{k}=$ $\left\langle a_{l}-k+1, \ldots, a_{l}\right\rangle$ apart from the first one, and thus induces $h_{\varpi_{1}}(\varepsilon)$ on the subgroup of type $\Delta=\mathrm{B}_{k}, 1 \leq k \leq l-1$. Finally, $T_{\mathrm{sc}}\left(\mathrm{D}_{l}, K\right)=T_{\mathrm{sc}}\left(\mathrm{B}_{l}, K\right)$, so that the answer for $\Delta=\mathrm{D}_{k}$ is the same as for $\Phi=\mathrm{D}_{l}$.

- The system $\Phi=\mathrm{C}_{l}$ has the following proper irreducible subsystems: $\mathrm{A}_{k}, 1 \leq k \leq$ $l-1$, and $\mathrm{C}_{k}, 1 \leq k \leq l-1$. Clearly, $h_{\alpha_{l}}(\varepsilon)$ induces on $G\left(\mathrm{~A}_{l-1}, K\right)$ the diagonal automorphism corresponding to $h_{\varpi_{l-1}}(\varepsilon)$, which generates the entire group of diagonal automorphisms modulo the inner ones. However, $h_{\alpha_{k-1}}(\varepsilon)$ gives nothing new on $\mathrm{C}_{k}$.

- The system $\Phi=\mathrm{D}_{l}$ has the following proper irreducible subsystems: $\mathrm{A}_{k}, 1 \leq k \leq$ $l-1$, and $\mathrm{D}_{k}, 1 \leq k \leq l-1$. We have no need to worry about $\mathrm{A}_{k}, 1 \leq k \leq l-2$. Now, if $l$ is even, then $\Phi$ has two conjugacy classes of subsystems of type $\Delta=\mathrm{A}_{l-1}$, namely, $A_{l-1}^{\prime}=\left\langle\alpha_{1}, \ldots, \alpha_{l-2}, \alpha_{l-1}\right\rangle$ and $A_{l-1}^{\prime \prime}=\left\langle\alpha_{1}, \ldots, \alpha_{l-2}, \alpha_{l}\right\rangle$. In each case, the fundamental root of $\mathrm{D}_{l}$ not belonging to $\Delta$ induces the diagonal automorphism corresponding to $h_{\varpi_{l-2}}(\varepsilon)$. Finally, if $\Delta=\mathrm{D}_{k}=\left\langle\alpha_{l-k+1}, \ldots, \alpha_{l}\right\rangle$, then $h_{\alpha_{l-k}}(\varepsilon)$ induces on $G(\Delta, K)$ the same diagonal automorphism as $h_{\varpi_{1}}(\varepsilon)$.

- The system $\Phi=\mathrm{E}_{6}$ has the following proper irreducible subsystems: $\mathrm{A}_{k}, 1 \leq$ $k \leq 5 ; \mathrm{D}_{5} ; \mathrm{D}_{4}$. Everything is clear for $\mathrm{A}_{k}, 1 \leq k \leq 4$. Now, $\mathrm{A}_{5}=\left\langle\alpha_{1}, \alpha_{3}, \ldots, \alpha_{6}\right\rangle$. Clearly, $h_{\alpha_{2}}(\varepsilon)$ induces the same diagonal automorphism on $\mathrm{A}_{5}$ as $h_{\varpi_{3}}(\varepsilon)$. If $\Delta=$ $\mathrm{D}_{5}=\left\langle\alpha_{1}, \ldots, \alpha_{5}\right\rangle$, then $h_{\alpha_{2}}(\varepsilon)$ induces the same diagonal automorphism on $G(\Delta, K)$ as $h_{\varpi_{5}}(\varepsilon)$, which generates the entire group of diagonal automorphisms modulo the inner ones. Finally, if $\Delta=\mathrm{D}_{4}=\left\langle\alpha_{2}, \ldots, \alpha_{5}\right\rangle$, then $h_{\varpi_{1}}(\varepsilon)$ is induced already in $\mathrm{D}_{5}$, and $h_{\varpi_{4}}(\varepsilon)$ is induced by $h_{\alpha_{6}}(\varepsilon)$, as above.

- The system $\Phi=E_{7}$ has the following proper irreducible subsystems: $\mathrm{A}_{k}, 1 \leq k \leq 7$; $\mathrm{D}_{k}, 4 \leq k \leq 6 ; \mathrm{E}_{6}$. The subsystems of type $\mathrm{A}_{5}$ fall into two conjugacy classes: those of class $\mathrm{A}_{5}^{\prime}$ are contained in a subsystem of type $\mathrm{A}_{7}$ and those of class $\mathrm{A}_{5}^{\prime \prime}$ are not. For systems of types $\mathrm{A}_{k}, k=1,2,3,4,6$, and for $\mathrm{A}_{5}^{\prime}$ all diagonal automorphisms are already induced in $\mathrm{A}_{7}$. A subsystem $\Delta=\mathrm{A}_{7}$ may be generated by all fundamental roots $\alpha_{i}$, $i \neq 2$, and the negative highest root $\alpha_{0}$. The only fundamental root $\alpha_{2}$ that is not in $\Delta$ induces on $G(\Delta, K)$ the same diagonal automorphism as $h_{\varpi_{4}}(\varepsilon)$, the corresponding group having index 4 in the group of all diagonal automorphisms. Next, a subsystem $\Delta=\mathrm{A}_{5}^{\prime \prime}$ may be generated by $\alpha_{2}, \alpha_{4}, \ldots, \alpha_{7}$. The element $h_{\alpha_{3}}(\varepsilon)$ induces the same diagonal automorphism on $\Delta$ as $h_{\varpi_{2}}(\varepsilon)$. Now, $\Delta=D_{6}=\left\langle\alpha_{2}, \ldots, \alpha_{7}\right\rangle$ and $h_{\alpha_{1}}(\varepsilon)$ 
induces the same diagonal automorphism on $G(\Delta, K)$ as $h_{\varpi_{l}}(\varepsilon)$, the corresponding group having index 2 in the group of all diagonal automorphisms. Together with the inner diagonal automorphisms of type $\mathrm{D}_{6}$, it induces all diagonal automorphisms for $\Delta=$ $\mathrm{D}_{4}, \mathrm{D}_{5}$. Finally, $h_{\alpha_{7}}(\varepsilon)$ induces $h_{\varpi_{6}}(\varepsilon)$ on the subgroup of type $\Delta=\mathrm{E}_{6}=\left\langle\alpha_{1}, \ldots, \alpha_{6}\right\rangle$.

- The system $\mathrm{E}_{8}$ has the following proper irreducible subsystems: $\mathrm{A}_{k}, 1 \leq k \leq 8$; $\mathrm{D}_{k}, 4 \leq k \leq 8 ; \mathrm{E}_{6}, \mathrm{E}_{7}$. The subsystems of type $\mathrm{A}_{7}$ fall into two conjugacy classes: those of class $\mathrm{A}_{7}^{\prime}$ are contained in a subsystem of type $\mathrm{A}_{8}$ and those of class $\mathrm{A}_{7}^{\prime \prime}$ are not. Everything is clear for the systems of types $\mathrm{A}_{k}, 1 \leq k \leq 6, \mathrm{~A}_{7}^{\prime}$, and $\mathrm{E}_{6}$. A subsystem $\Delta=\mathrm{A}_{8}$ may be generated by all fundamental roots $\alpha_{i}, i \neq 2$, and the negative highest root $\alpha_{0}$. The only fundamental root $\alpha_{2}$ that is not in $\Delta$ induces the same diagonal automorphism on $G(\Delta, K)$ as $h_{\varpi_{3}}(\varepsilon)$, the corresponding group having index 3 in the group of all diagonal automorphisms. Next, a subsystem $\Delta=\mathrm{A}_{7}^{\prime \prime}$ may be generated by $\alpha_{2}, \alpha_{4}, \ldots, \alpha_{8}, \alpha_{0}$. The element $h_{\alpha_{3}}(\varepsilon)$ induces the same diagonal automorphism on $\Delta$ as $h_{\varpi_{2}}(\varepsilon)$. Now $\Delta=\mathrm{D}_{6}=\left\langle\alpha_{2}, \ldots, \alpha_{8}, \alpha_{0}\right\rangle$, and $h_{\alpha_{1}}(\varepsilon)$ induces the same diagonal automorphism on $G(\Delta, K)$ as $h_{\varpi_{l}}(\varepsilon)$, the corresponding group having index 2 in the group of all diagonal automorphisms. Together with the inner diagonal automorphisms of type $\mathrm{D}_{8}$, it induces all diagonal automorphisms for $\Delta=\mathrm{D}_{k}, 1 \leq k \leq 7$. Finally, $h_{\alpha_{8}}(\varepsilon)$ induces $h_{\varpi_{7}}(\varepsilon)$ on the subgroup of type $\Delta=\mathrm{E}_{7}=\left\langle\alpha_{1}, \ldots, \alpha_{7}\right\rangle$.

- The system $\mathrm{F}_{4}$ has the following proper irreducible subsystems: $\mathrm{B}_{4}, \mathrm{D}_{4}, \mathrm{~A}_{3}, \mathrm{~B}_{3}, \mathrm{C}_{3}$, $\mathrm{A}_{2}, \widetilde{\mathrm{A}}_{2}, \mathrm{~B}_{2}, \mathrm{~A}_{1}, \widetilde{\mathrm{A}}_{1}$. All Chevalley subgroups of rank 4 have the same maximal torus as $\mathrm{F}_{4}$ itself. On the other hand, $\mathrm{A}_{2}$ and $\mathrm{A}_{1}$ may be embedded in $\mathrm{A}_{3} ; \widetilde{\mathrm{A}}_{1}$ and $\widetilde{\mathrm{A}}_{2}$ may be embedded in $\mathrm{C}_{3}$, and $\mathrm{B}_{3}, \mathrm{~B}_{2}$ may be embedded in $\mathrm{B}_{4}$, so that there is no problem for these subsystems. It remains to consider only the cases of $A_{3}$ and $C_{3}$. Since the torus does not change, the answer for the case $\mathrm{A}_{3}$ is the same as for the embeddings $\mathrm{A}_{3} \subseteq \mathrm{B}_{4}$ and $\mathrm{A}_{3} \subseteq D_{4}$. But for $\mathrm{C}_{3}=\left\langle\alpha_{2}, \alpha_{3}, \alpha_{4}\right\rangle$ the element $h_{\alpha_{1}}(\varepsilon)$ induces the same diagonal automorphism as $h_{\varpi_{3}}(\varepsilon)$, thus giving the entire group of diagonal automorphisms.

- Finally, for the $\mathrm{G}_{2}$ case there are three proper irreducible subsystems: $\mathrm{A}_{2}, \mathrm{~A}_{1}$, and $\widetilde{A}_{1}$. The torus for $G_{2}$ is the same as for $A_{2}$, so that nothing new is induced on a subgroup of type $A_{2}$. On the other hand, it is obvious that all diagonal automorphisms are induced on subgroups of types $\mathrm{A}_{1}$ and $\widetilde{\mathrm{A}}_{1}$.

This theorem suggests the easiest way to think of the extended simply connected Chevalley groups of types $\mathrm{E}_{6}$ and $\mathrm{E}_{7}$. Namely, they may be regarded as subgroups of the usual Chevalley groups $G_{\mathrm{sc}}\left(\mathrm{E}_{7}, K\right)$ and $G_{\mathrm{sc}}\left(\mathrm{E}_{8}, K\right)$, respectively. One could define these groups by

$$
\begin{aligned}
& \bar{G}_{\mathrm{sc}}\left(\mathrm{E}_{6}, K\right)=G_{\mathrm{sc}}\left(\mathrm{E}_{6}, K\right) \cdot T_{\mathrm{sc}}\left(\mathrm{E}_{7}, K\right), \\
& \bar{G}_{\mathrm{sc}}\left(\mathrm{E}_{7}, K\right)=G_{\mathrm{sc}}\left(\mathrm{E}_{7}, K\right) \cdot T_{\mathrm{sc}}\left(\mathrm{E}_{8}, K\right),
\end{aligned}
$$

respectively. Unfortunately, it is impossible to construct extended Chevalley groups of types $\mathrm{C}_{l}$ and $\mathrm{D}_{l}$ in a such a straightforward way.

\section{§10. Abelian unipotent Radicals}

In this and the following two sections we study Borel orbits of Levi factors for the case of parabolic subgroups with Abelian unipotent radicals in their conjugation action on these radicals.

Let $\omega=\varpi_{i}$ be a microweight of the dual root system $P\left(\Phi^{\vee}\right)$, and let

$$
\Sigma=\Sigma_{\omega}=\{\alpha \in \Phi \mid(\alpha, \omega)>0\}
$$

be as in the Introduction. Since $\omega$ is a microweight, the coefficient of $\alpha_{i}$ in the expansion of the highest root with respect to the fundamental system equals 1 . Thus, $\Sigma_{\omega}$ consists of 
all roots $\alpha \in \Phi$ such that the coefficient of $\alpha_{i}$ in their expansion as a linear combination of the fundamental roots equals 1 .

We consider this set as a partially ordered set with respect to the usual order defined by a choice of the fundamental system. In other words, $\alpha \succeq \beta$ if and only if $\alpha-\beta=\sum m_{i} \alpha_{i}$, where $m_{i} \geq 0$ for all $1 \leq i \leq l$. Consider the Hasse diagram of this poset. In other words, the vertices of this diagram correspond to the roots of $\Sigma_{\omega}$, and two vertices $\alpha$ and $\beta$ are joined by a bond if their difference is a fundamental root $\alpha_{i}$. In this case we mark this bond with $i$. As in many other works in which these diagrams are employed, our positive direction is from right to left and from bottom to top. In other words, a bond marked with $i$ when read from left to right expresses subtraction and not addition of $\alpha_{i}$. Another convention is that we omit the marks that may be restituted using the fact that the marks on the opposite sides of a parallelogram coincide.

Next, let $\Delta=\Delta_{\omega}$ be the closure of the set $\Pi \backslash\left\{\alpha_{i}\right\}$. In other words, $\Delta_{\omega}=\{\alpha \in \Phi \mid$ $(\alpha, \omega)=0\}$. Thus, if the difference of two roots in $\Sigma_{\omega}$ is a root, it must belong to $\Delta_{\omega}$. The diagram of the set $\Sigma_{\omega}$ coincides with the weight diagram of some representation of the Chevalley group $G\left(\Delta_{\omega}, K\right)$ of type $\Delta_{\omega}$ (see, for example, [73] and the references in [76, [58, 80]). Indeed, since the set $\Sigma_{\omega}$ is Abelian, the Chevalley commutator formula shows that the group $E\left(\Sigma_{\omega}\right)$ is Abelian. In fact, this group is precisely the unipotent radical of the maximal parabolic subgroup $Q_{i}=\bar{G}\left(\Delta_{\omega} \cup \Sigma_{\omega}\right)$. In particular, it is invariant under conjugation by elements of the Levi factor of $Q_{i}$. The group $G\left(\Delta_{\omega}, K\right)$ is a semisimple subgroup of this Levi factor and thus acts on $E\left(\Sigma_{\omega}\right)$ by conjugation:

$$
y \mapsto x y x^{-1}, x \in G\left(\Delta_{\omega}, K\right), y \in E\left(\Sigma_{\omega}\right) .
$$

For all pairs $(\Phi, \omega)$, in Table 3 we list the type of $\Delta=\Delta_{\omega}$, the highest weight $\rho$, and the dimension $n$ of this representation.

\section{TABLE 3}

$\begin{array}{lllll}\Phi & \omega & \Delta & \rho & n \\ \mathrm{~A}_{l} & \varpi_{k} & \mathrm{~A}_{k}+\mathrm{A}_{l-k-1} & \left(\varpi_{1}, \varpi_{1}\right) & (k+1)(l-k) \\ \mathrm{B}_{l} & \varpi_{1} & \mathrm{~B}_{l-1} & \varpi_{1} & 2 l-1 \\ \mathrm{C}_{l} & \varpi_{l} & \mathrm{~A}_{l-1} & 2 \varpi_{1} & l(l+1) / 2 \\ \mathrm{D}_{l} & \varpi_{1} & \mathrm{D}_{l-1} & \varpi_{1} & 2(l-1) \\ \mathrm{D}_{l} & \varpi_{l-1}, \varpi_{l} & \mathrm{~A}_{l-1} & \varpi_{2} & l(l-1) / 2 \\ \mathrm{E}_{6} & \varpi_{1}, \varpi_{6} & \mathrm{D}_{5} & \varpi_{5} & 16 \\ \mathrm{E}_{7} & \varpi_{7} & \mathrm{E}_{6} & \varpi_{1} & 27\end{array}$

In what follows, we view $E\left(\Sigma_{\omega}\right)$ as a vector space $V$ of dimension $n$ with a base $v^{\gamma}$ labeled by the roots $\gamma \in \Sigma_{\omega}$. In other words, addition in $V$ is defined by multiplication in $E(\Sigma, K)$, and scalar multiplication by $\xi \in K$ in $V$ corresponds to the simultaneous multiplication by $\xi$ of all coefficients $a_{\gamma}$ in the expression $v=\prod x_{\gamma}\left(a_{\gamma}\right), \gamma \in \Sigma_{\omega}$, of an element $v \in E(\Sigma, K)$ in terms of elementary root unipotents. We can choose any nontrivial elements $x_{\gamma}(*) \in X_{\gamma}$ as such a base, for example, the elements $v^{\gamma}=x_{\gamma}(1)$. As we have seen above, this $V$ affords a representation of the Chevalley group of type $\Delta$. With the exceptions of $\mathrm{B}_{l}$ and $\mathrm{C}_{l}$, this representation is itself a microweight representation, 
and the action of the unipotent root elements $x_{\alpha}(\xi), \alpha \in \Delta, \xi \in K$, is expressed as follows:

$$
x_{\alpha}(\xi) v^{\gamma}= \begin{cases}v^{\gamma} & \text { if } \gamma+\alpha \notin \Sigma_{\omega}, \\ v^{\gamma} \pm \xi v^{\gamma+\alpha} & \text { if } \gamma+\alpha \in \Sigma_{\omega} .\end{cases}
$$

This is precisely the Chevalley commutator formula for these cases. It is also worthwhile to compare this with Matsumoto's lemma [54, 73, 76. In the cases of $\mathrm{B}_{l}$ and $\mathrm{C}_{l}$, the resulting representation is not a microweight one, and the formulas may become slightly more complicated. Observe, though, that for the $\mathrm{B}_{l}$ case this representation is still basic in the sense of Matsumoto 34. In the second formula the coefficient 2 appears if both $\alpha$ and $\gamma$ are short roots; this is precisely why we must exclude fields of characteristic 2 for these cases. On the other hand, when $\alpha$ is short but $\gamma$ is long, the formula becomes

$$
x_{\alpha}(\xi) v^{\gamma}=v^{\gamma} \pm \xi v^{\gamma+\alpha} \pm \xi^{2} v^{\gamma+2 \alpha} .
$$

This action may be visualized in terms of the above diagram. Conceive a vector $v \in V$ as a marked graph obtained by putting marks $c_{\lambda}$ and $c_{\alpha}^{0}$ at the corresponding vertices of the weight diagram of type $(\Delta, \rho)$. Expand a root $\alpha \in \Delta$ with respect to the fixed base of the root system: $\alpha=\sum m_{i} \alpha_{i}, \alpha_{i} \in \Pi(\Delta)$. Then $x_{\alpha}(\xi)$ acts on $v$ as follows. Assume that there is a directed path from $\gamma$ to $\delta$, having precisely $\left|m_{i}\right|$ bonds with mark $i$ for all $i$ appearing in the expression of $\alpha$. Recall that we go in the positive/negative direction if $m_{i}$ are positive/negative. Then $x_{\alpha}(\xi)$ multiplies the coordinate of $v$ standing at the vertex $\gamma$ by $\pm \xi$ and adds it to the coordinate standing at the vertex $\delta$. There are slightly more complicated rules if there are two paths with the same set of marks that start/stop at the same vertex. In those cases we multiply some coordinates by $\pm 2 \xi$ or $\pm \xi^{2}$. See [73, 76] and the references therein for a description of calculations with such diagrams and many further references. Below we use diagrams to visualize our calculations.

\section{§11. Orbits of the Borel unipotent RADical}

Now we pass to the study of Borel orbits for the cases listed in Table 3. We start with the orbits of the unipotent radical $U(\Delta, K)$. These are precisely the representations that arise in the proof of the main theorem.

Proposition 2. Let $(V, \rho)$ be one of the representations listed in Table 3. Suppose that char $K \neq 2$ if either $\Delta=\mathrm{B}_{l-1}$ and $\rho=\varpi_{1}$, or $\Delta=\mathrm{A}_{l-1}$ and $\rho=2 \varpi_{1}$. Then each orbit of $U=U(\Delta, K)$ on $V$ contains a vector $v=\sum a_{\gamma} v_{\gamma}$ whose support $S(v)=\{\gamma \in$ $\left.\Sigma_{\omega} \mid a_{\gamma} \neq 0\right\}$ consists of mutually strictly orthogonal roots. Any two such vectors lie in distinct $U$-orbits.

Proof. We analyse all arising representations case by case. To slightly simplify notation, we study orbits of the unipotent radical $U^{-}=U^{-}(\Delta, K)$ of the opposite Borel subgroup $B^{-}(\Delta, K)$. Symmetry with respect to the positive and negative roots guarantees that the answer carries over to the Borel subgroup $B(\Delta, K)$ itself.

- First, let $\Delta=\mathrm{A}_{k}+\mathrm{A}_{l-k-1}, \rho=\left(\varpi_{1}, \varpi_{1}\right)$. Then the corresponding poset $\Sigma=\Sigma_{\omega}$ has the diagram depicted in Figure 1.

We prove the proposition by induction on $\min (k, l-k-1)$. Due to symmetry of the factors, without loss of generality we may assume that $k \leq l-k-1$. Take any vector $v=\sum a_{\gamma} v^{\gamma}$ in $V$. If $v=0$, there is nothing to prove.

First, let $k=0$. Then the corresponding poset is a chain, and all its bonds have distinct marks. Look at the largest root $\delta$ such that $a_{\delta} \neq 0$. If $\gamma \succ \delta$, then, by the definition of $\delta$, we have $a_{\gamma}=0$. On the other hand, if $\gamma \prec \delta$, then $\alpha=\gamma-\delta$ is a negative root in $\Delta$. Thus, we can multiply $v$ by $x_{\alpha}\left( \pm a_{\gamma} / a_{\delta}\right) \in U^{-}(\Delta, K)$ and kill the coordinate with label $\gamma$, without changing any other coordinate. Doing this, for all $\gamma \prec \delta$ we get a 


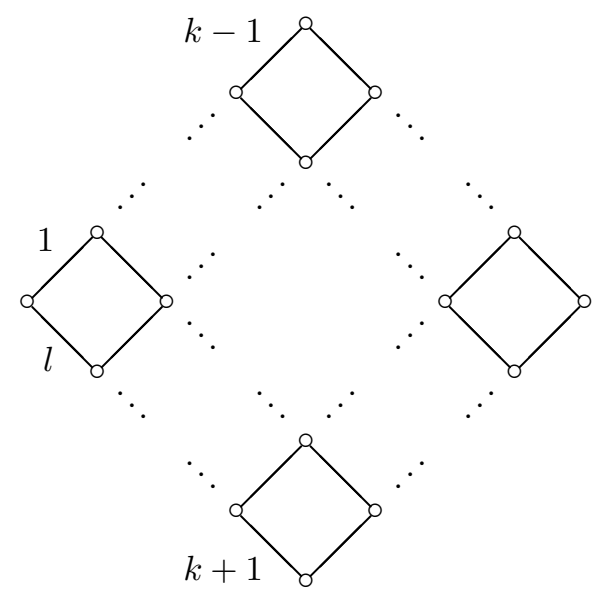

FiguRE 1

vector lying in the $U^{-}$-orbit of $v$ and such that all its coordinates are zeros except for the coordinate with label $\delta$.

Now, let $k>0$. Suppose we have already proved the proposition for the system $\Delta^{\prime}=\mathrm{A}_{k-1}+\mathrm{A}_{l-k-1}$. First, let $\delta$ be the highest root. Look at the coordinates $a_{\gamma}$, $\gamma \in \Theta=\left\{\delta, \delta-\alpha_{1}, \ldots, \delta-\alpha_{1}-\cdots-\alpha_{k}\right\}$. If all of them are zeros, then we are done by the inductive hypothesis. Suppose that some of these coordinates are not zero. Let $\delta$ be the largest root in $\Theta$ such that $a_{\delta} \neq 0$. Let $\Omega$ be the set of roots in $\Sigma_{\omega}$ orthogonal to $\delta$. The poset $\Sigma \backslash \Omega$ is a join of two chains with a unique common point $\delta$. All marks on the bonds of these two chains are distinct. If $\gamma \in \Sigma \backslash \Omega$, then $\gamma-\delta$ is a negative root in $\Delta$. By the definition of $\delta$, we have $a_{\gamma}=0$ for all $\gamma \prec \delta$. Now, if $\gamma \in \Sigma \backslash \Omega, \gamma \succ \delta$, then we can multiply $v$ by $x_{\alpha}\left( \pm a_{\gamma} / a_{\delta}\right) \in U^{-}(\Delta, K)$ and kill the coordinate with label $\gamma$ without changing other coordinates with labels belonging to $\Sigma \backslash \Omega$. Repeating this procedure we may assume that the only nonzero coordinates, apart from the coordinate with label $\delta$, have labels belonging to $\Omega$.

Now, $\Omega$ has the same structure as $\Sigma$, but has one less column and one less row. Namely, the space $V^{\prime}=\left\langle v^{\gamma}, \gamma \in \Omega\right\rangle$ affords the representation with the highest weight $\left(\varpi_{1}, \varpi_{1}\right)$ for the root subsystem $\Delta^{\prime}=\mathrm{A}_{k-1}+\mathrm{A}_{l-k-2}$ generated by all fundamental roots $\alpha_{i} \in \Delta$ such that $\delta \pm \alpha_{i}$ is not a root, and by the root $\alpha^{\prime}=\alpha_{h}+\alpha_{h+1}$, where $\alpha_{h}$ is such that $\delta-\alpha_{h}$ is a root if $h \neq l-k-1, l$. In the preceding paragraph we established that by transformations belonging to $U^{-}$our vector $v$ may be reduced to the form $\xi v^{\delta}+v^{\prime}$ with some $\xi \in K^{*}, v^{\prime} \in V^{\prime}$. It only remains to apply the inductive hypothesis to $v^{\prime}$ and to observe that the roots $\alpha_{i}$ such that $\delta \pm \alpha_{i}$ is not a root, and also the root $\alpha^{\prime}$, are all orthogonal to $\delta$, so that any additions in $V^{\prime}$ stabilize $v^{\delta}$.

- Let $\Delta=\mathrm{B}_{l-1}, \rho=\varpi_{1}$. Then the corresponding poset $\Sigma=\Sigma_{\omega}$ has the diagram as in Figure 2.

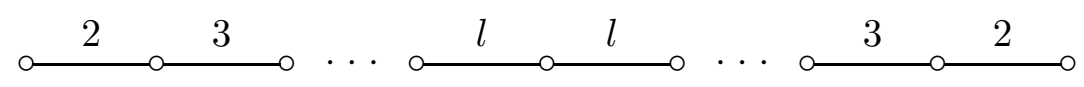

FiguRE 2 
Take any vector $v=\sum a_{\gamma} v^{\gamma}$ in $V$. For $v=0$ there is nothing to prove. Let $\delta$ be the largest root such that $a_{\delta} \neq 0$. We distinguish two cases.

First, suppose that $\delta$ is long. Then there is precisely one root $\delta^{*}$ in $\Sigma$ orthogonal to $\delta$. By the definition of $\delta$, we have $a_{\gamma}=0$ for any root $\gamma \succ \delta$. Now, let $\gamma \prec \delta, \gamma \neq \delta^{*}$. Then $\alpha=\gamma-\delta$ is a negative root in $\Delta$. Thus, we can multiply $v$ by $x_{\alpha}\left( \pm a_{\gamma} / a_{\delta}\right) \in U^{-}(\Delta, K)$ and kill the coordinate with label $\gamma$. Doing this, we do not change any other coordinate, apart possibly from that with label $\delta^{*}$. Repeating this procedure, we may assume that all the coordinates of $v$ are zeros except for the coordinates with labels $\delta, \delta^{*}$. On the other hand, we have no control over the coordinate with label $\delta^{*}$. Indeed, let $($,$) be the usual$ inner product on $V$ invariant under the action of $G(\Delta, K)$. Should the initial vector $v$ be anisotropic, it would remain anisotropic after reduction with elements of $U^{-}(\Delta, K)$. But the scalar square of a vector with only two nonzero coordinates $a_{\delta}$ and $a_{\delta^{*}}$ equals $2 a_{\delta} a_{\delta^{*}}$.

Now, let $\delta$ be short. Then there are no roots orthogonal to $\delta$, and by the definition of $\delta$ we have $a_{\gamma}=0$ for all $\gamma \succ \delta$. For any $\gamma \prec \delta$ the difference $\alpha=\gamma-\delta$ is a root, and multiplying $v$ by $x_{\alpha}\left( \pm a_{\gamma} / 2 a_{\delta}\right)$ we may kill the coordinate with label $\gamma$ without changing any other coordinate. Recall that char $K \neq 2$ in this case! Repeating this procedure, we get a vector whose only nonzero coordinate is that with label $\delta$.

- Let $\Delta=\mathrm{A}_{l-1}, \rho=2 \varpi_{1}$. The diagram of the corresponding $\Sigma=\Sigma_{\omega}$ is depicted in Figure 3.

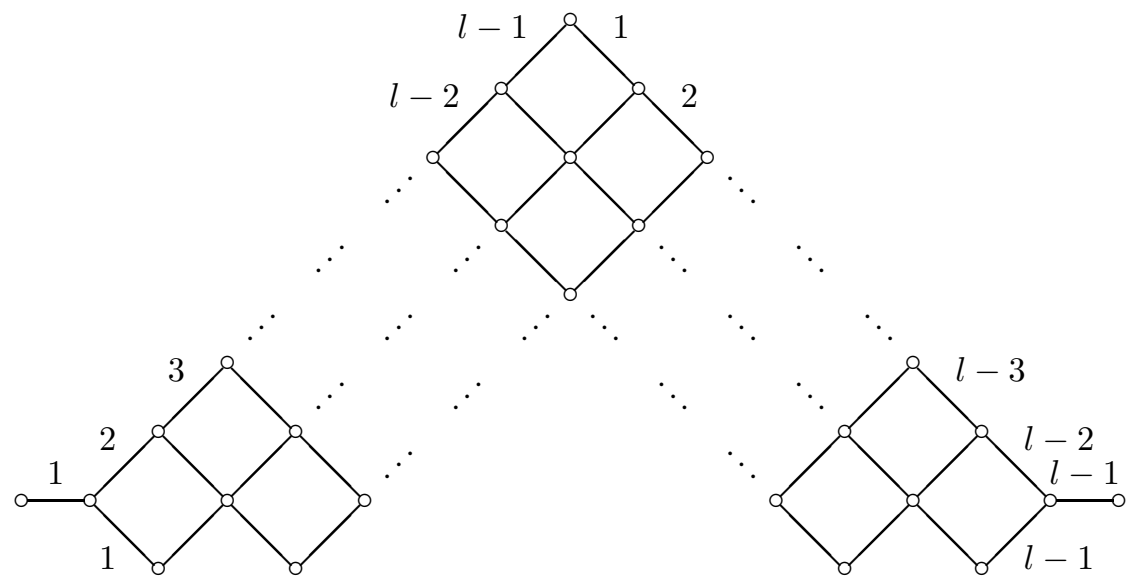

Figure 3

We prove the proposition by induction on $l$. If $l \leq 2$, we fall into the situation considered in the preceding case. Now, assume that $l \geq 3$ and that the proposition is already proved for the pairs $\left(A_{k}, 2 \varpi_{1}\right)$ for all $k<l-1$. Take any vector $v=\sum a_{\gamma} v^{\gamma}$ in $V$. If $v=0$, then there is nothing to prove. First, let $\delta$ be the highest root. Look at the coordinates $a_{\gamma}, \gamma \in \Theta=\left\{\delta, \delta-\alpha_{1}, \ldots, \delta-\alpha_{1}-\cdots-\alpha_{l-1}\right\}$. If all of these coordinates are zeros, then we are done by the inductive hypothesis. Suppose some of these coordinates are not zero. Let $\delta$ be the largest root in $\Theta$ such that $a_{\delta} \neq 0$. Now we can argue exactly as in the case of $\Delta=A_{k}+A_{l-k-1}, \rho=\left(\varpi_{1}, \varpi_{1}\right)$. Let $\Omega$ be the set of roots in $\Sigma_{\omega}$ orthogonal to $\delta$. At this point we need to distinguish two cases.

If $\delta$ is long, then it is the highest root and the poset $\Sigma \backslash \Omega$ is a chain. All the marks on the bonds of this chain are distinct. If $\gamma \in \Sigma \backslash \Omega$, then $\gamma-\delta$ is a negative root in 
$\Delta$. Now, for any $\gamma \in \Sigma \backslash \Omega, \gamma \prec \delta$, we can multiply $v$ by $x_{\alpha}\left( \pm a_{\gamma} / a_{\delta}\right) \in U^{-}(\Delta, K)$ and kill the coordinate with label $\gamma$ without changing any other coordinate with labels in $\Sigma \backslash \Omega$. Repeating this procedure, we may assume that the only nonzero coordinates have labels from $\Omega$, apart from the coordinate with label $\delta$. Now, $\Omega$ is of the same structure as $\Sigma$, but has one less column and one less row. Namely, the space $V^{\prime}=\left\langle v^{\gamma}, \gamma \in \Omega\right\rangle$ affords the representation with the highest weight $2 \varpi_{1}$ for the root subsystem $\Delta^{\prime}=\mathrm{A}_{l-2}$ generated in $\Delta$ by all fundamental roots $\alpha_{i}$ except for $\alpha_{1}$. We have proved above that by transformations belonging to $U^{-}$our vector $v$ may be reduced to the form $\xi v^{\delta}+v^{\prime}$, for some $\xi \in K^{*}, v^{\prime} \in V^{\prime}$. It only remains to apply the inductive hypothesis to $v^{\prime}$ and to observe that all roots $\alpha_{i}, i \geq 2$, are orthogonal to $\delta$, so that any additions in $V^{\prime}$ stabilize $v^{\delta}$.

Life is somewhat more complicated when $\delta$ is short. In this case the structure of the poset $\Xi=\{\gamma \in \Sigma \backslash \Omega \mid \gamma \prec \delta\}$ is as in Figure 4,

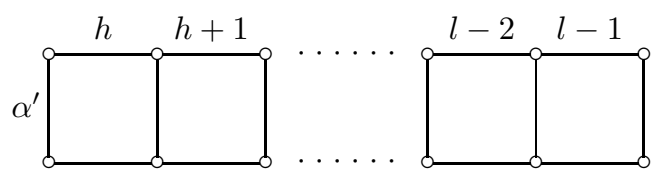

FiguRE 4

where $\alpha_{h} \neq \alpha_{1}$ is the fundamental root of $\Delta$ such that $\delta-\alpha_{h}$ is a root and $\alpha^{\prime}=$ $\alpha_{1}+\cdots+\alpha_{h-1}$. If there is no such root $\alpha_{h}$, then we must set $\alpha^{\prime}=\alpha_{1}+\cdots+\alpha_{l-1}$. If $\gamma \in \Xi$, then $\gamma-\delta$ is a negative root in $\Delta$. Now, for any $\gamma \in \Xi$ we can multiply $v$ by $x_{\alpha}\left( \pm a_{\gamma} / a_{\delta}\right)$ if $\gamma$ is short and by $x_{\alpha}\left( \pm a_{\gamma} / 2 a_{\delta}\right)$ if $\gamma$ is long and kill the coordinate with label $\gamma$ without changing other coordinates with labels in $\Xi$. Repeating this procedure, we may assume that the only nonzero coordinates have labels in $\Omega$, apart from the coordinate with label $\delta$. Now, $\Omega$ is of the same structure as $\Sigma$, but has two fewer columns and two fewer rows. The space $V^{\prime}=\left\langle v^{\gamma}, \gamma \in \Omega\right\rangle$ affords the representation with the highest weight $2 \varpi_{1}$ for the root subsystem $\Delta^{\prime}=\mathrm{A}_{l-3}$ generated in $\Delta$ by all fundamental roots $\alpha_{i}$ except for $\alpha_{1}, \alpha_{h-1}, \alpha_{h}$, and by the root $\alpha^{\prime \prime}=\alpha_{h-1}+\alpha_{h}$. We have proved that, by transformations belonging to $U^{-}$, our vector $v$ may be reduced to the form $\xi v^{\delta}+v^{\prime}$, for some $\xi \in K^{*}, v^{\prime} \in V^{\prime}$. It only remains to apply the inductive hypothesis to $v^{\prime}$ and to observe that the roots $\alpha_{i}, i \neq 1, h-1, h$, and the root $\alpha^{\prime \prime}$ are orthogonal to $\delta$, so that any additions in $V^{\prime}$ stabilize $v^{\delta}$.

- Let $\Delta=\mathrm{D}_{l-1}, \rho=\varpi_{1}$. The corresponding $\Sigma=\Sigma_{\omega}$ has a diagram as in Figure 5 .

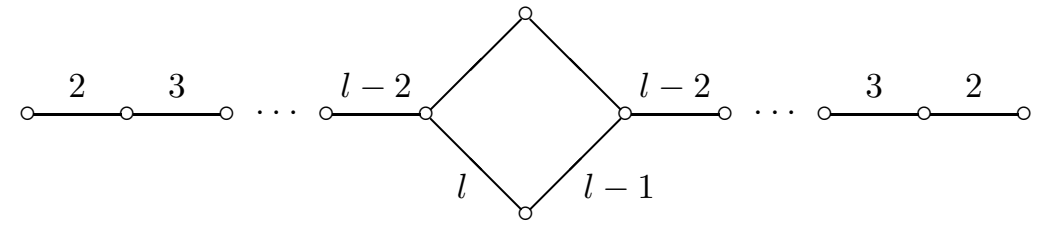

FiguRE 5

This case is settled by precisely the same argument as the case where $\Delta=\mathrm{B}_{l-1}, \rho=\varpi_{1}$. It is even easier, because there are no short roots here and only the first half of the proof should be repeated. 
- Let $\Delta=\mathrm{A}_{l-1}, \rho=\varpi_{2}$. The diagram of the corresponding $\Sigma=\Sigma_{\omega}$ is depicted in Figure 6.

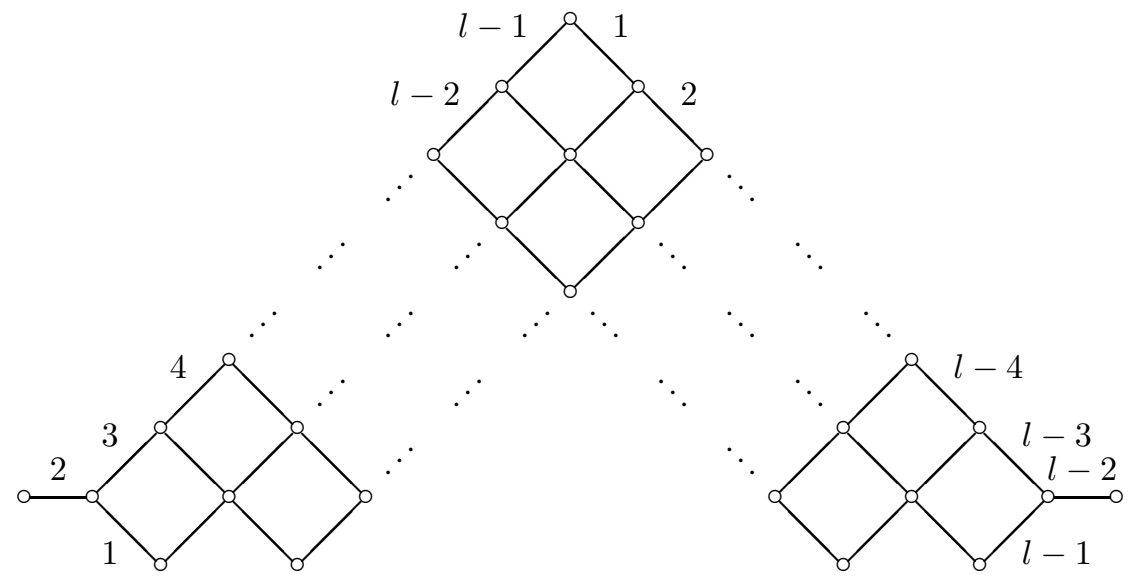

FiguRE 6

This case is settled by basically the same arguments as the case where $\Delta=\mathrm{A}_{l-1}$, $\rho=2 \varpi_{1}$. It is even somewhat easier because there are no short roots here. Or, rather, all roots behave like short roots, so that we only need to reproduce the second part of the induction step. Again, we prove the proposition by induction on $l$. If $l=2$, then there is nothing to prove. Now, assume that $l \geq 3$ and that the proposition is already proved for the pairs $\left(A_{k}, \varpi_{2}\right)$ for all $k<l-1$. Take any vector $v=\sum a_{\gamma} v^{\gamma}$ in $V$. For $v=0$ there is nothing to prove. First, let $\delta$ be the highest root. Look at the coordinates $a_{\gamma}$, $\gamma \in \Theta=\left\{\delta, \delta-\alpha_{2}, \ldots, \delta-\alpha_{2}-\cdots-\alpha_{l-1}\right\}$. If all of these coordinates are zeros, then we are done by the inductive hypothesis. Suppose some of these coordinates are not zero. Now, let $\delta$ be the largest root in $\Theta$ such that $a_{\delta} \neq 0$. Let $\Omega$ be the set of roots in $\Sigma_{\omega}$ orthogonal to $\delta$. Consider the poset $\Xi=\{\gamma \in \Sigma \backslash \Omega \mid \gamma \prec \delta\}$. It consists of the roots $\gamma=\delta-\alpha^{\prime}+\alpha_{h-1}, \delta-\alpha_{h}, \ldots, \delta-\alpha_{h}-\cdots-\alpha_{l-1}, \delta-\alpha^{\prime}-\alpha_{h}, \ldots, \delta-\alpha^{\prime}-\alpha_{h}-\cdots-\alpha_{l-1}$. Multiplying $v$ by $x_{\alpha}\left( \pm a_{\gamma} / a_{\delta}\right)$, where $\alpha=\gamma-\delta$ is a negative root in $\Delta$, we can kill the coordinate of $v$ with label $\gamma$. Repeating this procedure, we may assume that the only nonzero coordinates have labels in $\Omega$, apart from the coordinate with label $\delta$. Now, we can repeat the arguments for the case of $\Delta=\mathrm{A}_{l-1}, \rho=2 \varpi_{1}$ word for word. Again, $\Omega$ has the same structure as $\Sigma$, but has two fewer columns and two fewer rows. The space $V^{\prime}=\left\langle v^{\gamma}, \gamma \in \Omega\right\rangle$ affords the representation with the highest weight $\varpi_{2}$ for the root subsystem $\Delta^{\prime}=A_{l-3}$ generated in $\Delta$ by all the fundamental roots $\alpha_{i}$ except for $\alpha_{1}, \alpha_{h-1}, \alpha_{h}$, and the root $\alpha^{\prime \prime}=\alpha_{h-1}+\alpha_{h}$. We have proved that, by transformations belonging to $U^{-}$, our vector $v$ may be reduced to the form $\xi v^{\delta}+v^{\prime}$, for some $\xi \in K^{*}$, $v^{\prime} \in V^{\prime}$. It only remains to apply the inductive hypothesis to $v^{\prime}$ and to observe that the roots $\alpha_{i}, i \neq 1, h-1, h$, and also the root $\alpha^{\prime \prime}$, are all orthogonal to $\delta$, so that any additions in $V^{\prime}$ stabilize $v^{\delta}$.

- Let $\Delta=\mathrm{D}_{5}, \rho=\varpi_{5}$. The diagram of the corresponding $\Sigma=\Sigma_{\omega}$ is as in Figure 7 . Take any vector $v=\sum a_{\gamma} v^{\gamma}$ in $V$. For $v=0$ there is nothing to prove. Now, let $\delta$ be a maximal root such that $a_{\delta} \neq 0$. Multiplying $v$ by $x_{\alpha}\left( \pm a_{\gamma} / \alpha_{\delta}\right)$, where $\alpha=\gamma-\delta$, we may kill any coordinate with label $\gamma$ such that $\gamma$ is not orthogonal to $\delta$ and $\gamma \prec \delta$. Recall that all coordinates corresponding to $\gamma \succ \delta$ are zeros by the definition of $\delta$. Let $\Omega$ be the set of all roots $\gamma$ that are orthogonal to $\delta$, and let $V^{\prime}=\left\langle v^{\gamma}, \gamma \in \Omega\right\rangle$ be the corresponding subspace in $V$. We have proved that under the action of $U^{-}$any vector $v \in V$ is equivalent to a vector of the form $\xi v^{\delta}+v^{\prime}$, for some $v^{\prime} \in V^{\prime}$. But the set $\Omega$ of 


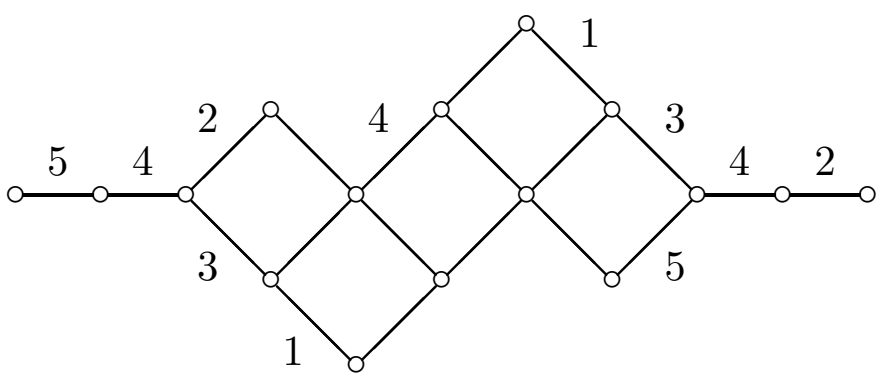

FiguRE 7

all roots $\gamma$ orthogonal to $\delta$ is a chain of length 5 affording the representation of a group of type $\Delta^{\prime}=\mathrm{A}_{4}$ with the highest weight $\varpi_{1}$. From the first case we know that under the action of $U^{-}\left(\Delta^{\prime}, K\right)$ such a vector can be reduced to the form $\zeta v^{\gamma}$ for some $\gamma \in \Omega$. Since all roots in $\Delta^{\prime}$ are orthogonal to $\delta$, this action of $U^{-}\left(\Delta^{\prime}, K\right)$ does not spoil the rest of the picture, and any vector in $V$ is equivalent to a vector of the form $\xi v^{\delta}+\zeta v^{\gamma}$ for some orthogonal roots $\gamma$ and $\delta$ and some $\xi, \zeta \in K$.

- Finally, let $\Delta=\mathrm{E}_{6}, \rho=\varpi_{1}$. The corresponding $\Sigma=\Sigma_{\omega}$ has the diagram as in Figure 8.

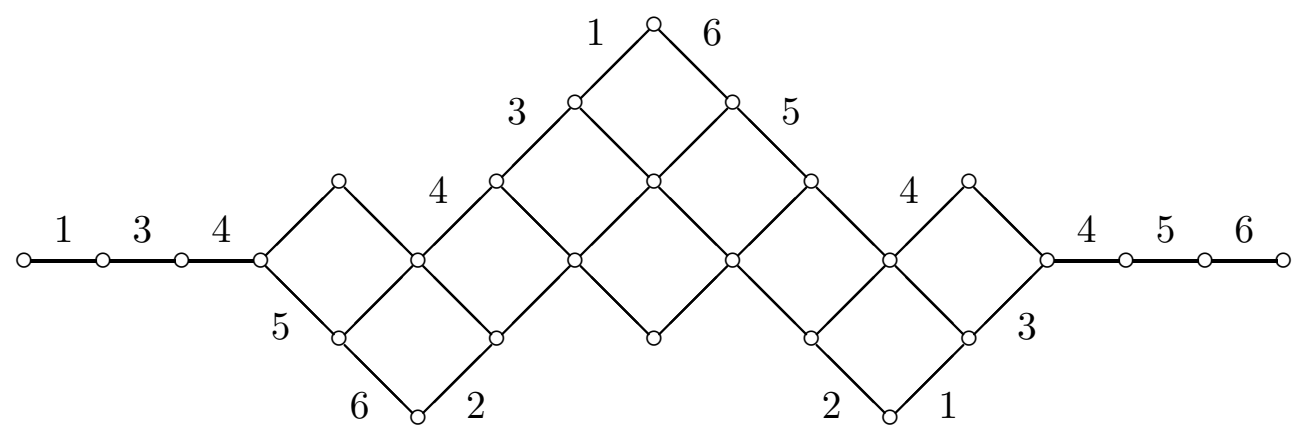

FiguRE 8

We argue as in the preceding case. Take any vector $v=\sum a_{\gamma} v^{\gamma}$ in $V$. For $v=0$ there is nothing to prove. Now, let $\delta$ be a maximal root such that $a_{\delta} \neq 0$. Multiplying $v$ by $x_{\alpha}\left( \pm a_{\gamma} / \alpha_{\delta}\right)$, where $\alpha=\gamma-\delta$, we may kill any coordinate with label $\gamma$ such that $\gamma$ is not orthogonal to $\delta$ and $\gamma \prec \delta$ - all coordinates corresponding to $\gamma \succ \delta$ are zeros by the definition of $\delta$. Let $\Omega$ be the set of all roots $\gamma$ orthogonal to $\delta$, and let $V^{\prime}=\left\langle v^{\gamma}, \gamma \in \Omega\right\rangle$ be the corresponding subspace in $V$. We have proved that under the action of $U^{-}$any vector $v \in V$ is equivalent to a vector of the form $\xi v^{\delta}+v^{\prime}$ for some $v^{\prime} \in V^{\prime}$. But the set $\Omega$ of all roots $\gamma$ orthogonal to $\delta$ forms the diagram of the representation of a group of type $\Delta^{\prime}=D_{5}$ with the highest weight $\varpi_{1}$. From the fourth case we know that under the action of $U^{-}\left(\Delta^{\prime}, K\right)$ such a vector can be reduced to the form $\zeta v^{\gamma}+\eta v^{\gamma^{*}}$ for some $\gamma \in \Omega$, where $g^{*}$ is determined by $\gamma$ as in the second case. Since all roots in $\Delta^{\prime}$ are orthogonal to $\delta$, these additions from $U^{-}\left(\Delta^{\prime}, K\right)$ do not influence coordinates with labels not belonging to $\Omega$, so that any vector in $V$ is equivalent to a vector of the form $\xi v^{\delta}+\zeta v^{\gamma}+\eta v^{\gamma^{*}}$ for some orthogonal roots $\delta, \gamma$ and $\gamma^{*}$ and some $\xi, \zeta, \eta \in K$.

This completes the proof of the proposition. 


\section{§12. BOREL ORBITS}

In this section we conclude the analysis of Borel orbits with respect to the extended Borel subgroup $\bar{B}(\Delta, K)$. Namely, we show that each element $v$ in the representations under study can be reduced to a linear combination of base vectors corresponding to a set of mutually orthogonal roots in $\Sigma$ with some very special coefficients. In the following proposition we set $\bar{B}(\Delta, K)=U(\Delta, K) \cdot \bar{T}(\Phi, K)$. In fact, in most cases any vector can be reduced to the desired form by an element of a smaller group, often $B(\Delta, K)$ itself. However, we do not use this slightly stronger fact in the proof of the main theorem.

Proposition 3. We keep the assumptions of Proposition 3 and let $\Theta$ be a set of representatives of $K^{*}$ modulo $K^{* 2}$.

- If $(\Delta, \rho) \neq\left(\mathrm{B}_{l-1}, \varpi_{1}\right),\left(\mathrm{A}_{l-1}, 2 \varpi_{1}\right)$, then each orbit of $\bar{B}=\bar{B}(\Delta, K)$ on $V$ contains a vector $v_{\Gamma}=\sum v_{\gamma}$ whose support $\Gamma=\left\{\gamma \in \Sigma_{\omega} \mid a_{\gamma} \neq 0\right\}$ consists of mutually strictly orthogonal roots.

- If $(\Delta, \rho)=\left(\mathrm{B}_{l-1}, \varpi_{1}\right),\left(\mathrm{A}_{l-1}, 2 \varpi_{1}\right)$, then each orbit of $\bar{B}=\bar{B}(\Delta, K)$ on $V$ contains a vector $v_{\Gamma}=\sum a_{\gamma} v_{\gamma}$ whose support $\Gamma=\left\{\gamma \in \Sigma_{\omega} \mid a_{\gamma} \neq 0\right\}$ consists of mutually strictly orthogonal roots and all $a_{\gamma} \in \Theta$. For all short roots and one more long root appearing in this expansion with nonzero coefficients we may take $a_{\gamma}=1$.

- Any two vectors considered in the above items lie in distinct $\bar{B}$-orbits.

Proof. Comparison of the lists in Table 3 and Proposition 2 shows that, with the only exception of $\mathrm{A}_{l-1}$ and $\mathrm{D}_{l-1}$ in $\mathrm{D}_{l}$, the group $T(\Phi, K)$ already induces all the diagonal automorphisms on $G(\Delta, K)$.

The last claim of the proposition is clear for the typical case - it follows already from Proposition 3 - and will follow from the considerations required to prove the second item in the exceptional cases.

We start with the first item. For the case of $\Delta=\mathrm{A}_{k}+\mathrm{A}_{l-k-1}$ everything is clear. Here the conjugates $h=h_{w \varpi_{1}}(\varepsilon)$ of $h_{\varpi_{1}}(\varepsilon)$ under the action of the Weyl group have the following properties.

- For every root $\gamma$ there is a conjugate $h$ such that $h x_{\gamma}(\xi) h^{-1}=x_{\gamma}(\varepsilon \xi)$ or, what is the same, $h v^{\gamma}=\varepsilon v^{\gamma}$.

- If $h$ does not commute with $x_{\gamma}(1)$, then $h$ commutes with $x_{\delta}(1)$ for all roots $\delta$ orthogonal to $\gamma$, or, what is the same, $h v^{\delta}=v^{\delta}$ for all such roots.

Now, let $v \in V$ be a vector whose support $S(v)$ consists of pairwise orthogonal roots. Then for every root $\gamma \in S(v)$ there exists $h$ such that the coordinate of $h v$ with label $\gamma$ is equal to 1 , while all other coordinates remain unchanged.

Consider the case where $\Delta=\mathrm{D}_{l-1}$. Here, it is more convenient to think directly in terms of $\bar{T}(\Phi, K)$. This means that, unlike other cases, $\varpi_{i}$ refers to the fundamental roots of $\Phi$ rather than those of $\Delta$. Proposition 3 implies that, by a transformation of class $U^{-}(\Delta, K)$, any vector $v \in V$ may be reduced to the form $v=\xi v^{\gamma}+\zeta v^{\gamma^{*}}$. Now, $h_{\varpi_{l}}\left(\xi \zeta^{-1}\right) h_{\varpi_{1}}\left(\xi^{-1}\right) v=v^{\gamma}+v^{\gamma^{*}}$.

If $\Delta=\mathrm{A}_{l-1}, \rho=\varpi_{2}$, then the desired effect is obtained easily by the conjugates $h=h_{w \varpi_{1}}(\varepsilon)$ of the first fundamental weight element $h_{\varpi_{1}}(\varepsilon)$ under the action of the Weyl group (they have the same properties as in the first case).

For the case where $\Delta=D_{5}, \rho=\varpi_{5}$, the desired elements exist already in $T(\Delta, K)$. Indeed, for any two orthogonal roots $\gamma, \delta \in \Sigma$ there exists a root $\alpha \in \Delta$ such that $\gamma \pm \alpha \notin \Sigma$ and one of the roots $\delta+\alpha$ or $\delta-\alpha$ belongs to $\Sigma$. This is obvious for the case where $\gamma$ is the highest root, and the general case follows by passing to its conjugates under $W(\Delta)$. Take $v=\xi v^{\gamma}+\zeta v^{\delta}$ as in Proposition 2. Multiplying $v$ by $h_{\beta}\left(\xi^{-1}\right)$ with an appropriate $\beta \in \Delta$, we may assume that $v=v^{\gamma}+\eta v^{\delta}$. It only remains to multiply $v$ by $h_{\alpha}\left(\eta^{\mp 1}\right)$, where $\alpha$ is the root defined above. 
The most interesting case is, of course, that of $\Delta=\mathrm{E}_{6}$. In fact, in this case our statement follows from the results of [53]. For completeness, we give a direct proof. Let $v=\xi v^{\delta}+\zeta v^{\gamma}+\eta v^{\gamma^{*}}$ be as in the preceding proposition. The action of the Weyl group $W\left(\mathrm{E}_{6}\right)$ on the set of 270 triples $\left(\delta, \gamma, \gamma^{*}\right)$ of this kind is transitive; compare [76, $\left.\S 7\right]$ or [80]. Thus, without loss of generality we may content ourselves with any one of them. Let, for example, $\delta$ be the highest root and $\gamma$ the largest root among the roots in $\Sigma$ orthogonal to $\delta$. This is exactly the distinguished triple considered in [76. An easy calculation shows that

$$
h_{\varpi_{1}}\left(\xi^{-1} \zeta^{-1} \eta^{-1}\right) h_{\alpha_{1}}\left(\xi^{2} \zeta \eta\right) h_{\alpha_{6}}(\xi \zeta) v=v^{\delta}+v^{\gamma}+v^{\gamma^{*}} ;
$$

see [76, §7] for a detailed description of the elements $h_{\varpi_{1}}(\varepsilon), h_{\alpha_{1}}(\varepsilon), h_{\alpha_{6}}(\varepsilon)$.

Now we pass to the second item. First, let $\Delta=\mathrm{B}_{l-1}$. Let $v=\xi v^{\gamma}$, where $\gamma \in \Sigma$, or $v=\xi v^{\gamma}+\zeta v^{\gamma^{*}}$, where $\gamma \in \Sigma$ is a long root, as in Proposition 2. Multiplying $v$ by $h_{\varpi_{1}}\left(\xi^{-1}\right)$, we may assume without loss of generality that $\xi=1$. Now, $h_{\gamma^{*}}(\varepsilon)$ multiplies $v^{\gamma^{*}}$ by $\varepsilon^{2}$ without changing $v^{\gamma}$, so that $\zeta$ can be changed by any element in its class modulo $K^{* 2}$, but we cannot go any further.

Finally, let $\Delta=A_{l-1}, \omega=2 \varpi_{1}$. As above, by Proposition 2 we may assume that $v=\sum a_{\gamma} v^{\gamma}$, where the support of $v$ consists of mutually strictly orthogonal roots. For any short root $\gamma$ there exists a long root $\alpha$ such that $h_{\alpha}(\varepsilon) v^{\gamma}=\varepsilon v^{\gamma}$ and $h_{\alpha}(\varepsilon) v^{\beta}=v^{\beta}$ for any root strictly orthogonal to $\gamma$. Thus, we may assume that $a_{\gamma}=1$ for all short roots $\gamma$. If $\gamma$ is long, then $h_{\gamma}(\varepsilon)$ multiplies $v^{\gamma}$ by $\varepsilon^{2}$ and leaves all other $v^{\delta}$ invariant. This means that we can reduce the coefficients $a_{\gamma}$ modulo $K^{* 2}$. Finally, multiplying $v$ by $h_{\varpi_{l}}(\varepsilon)$, we can make one of the coefficients $a_{\gamma}$ equal to 1 . Clearly, there are no further transformations in $T_{\mathrm{sc}}\left(\mathrm{C}_{l}, K\right)$.

\section{§13. Typical Bruhat decomposition of a Weight ElEMENT}

In this section we prove the last subsidiary result we need for the proof of Theorem 1. Since it holds for an arbitrary weight and is of independent interest, we produce a general statement.

Theorem 4. Let $\omega \in P\left(\Phi^{\vee}\right)$ and $x \in \bar{G}$. Then for all $\varepsilon \in K^{*}$ apart from a finite number of them, the weight elements $x h_{\omega}(\varepsilon) x^{-1}$ belong to the same Borel double coset $\bar{B} w \bar{B}=\bar{B} w_{0} \bar{B}$. Furthermore, $w_{0}$ is an involution.

Proof. First, observe that the second assertion immediately follows from the first. Indeed, for any $\varepsilon$ we have $x h_{\omega}\left(\varepsilon^{-1}\right) x^{-1}=x h_{\omega}(\varepsilon) x^{-1} \in \bar{B} w^{-1} \bar{B}$. The first assertion implies that for almost all values of $\varepsilon$ both $\varepsilon$ and $\varepsilon^{-1}$ belong to the same double coset $\bar{B} w_{0} \bar{B}$. Thus, for any $\varepsilon$ in general position, $x h_{\omega}\left(\varepsilon^{-1}\right) x^{-1}$ belongs both to $\bar{B} w_{0} \bar{B}$ and to $\bar{B} w_{0}^{-1} \bar{B}$. Now the uniqueness of the Weyl factor in the Bruhat decomposition implies that $w_{0}=w_{0}^{-1}$.

Next, observe that to prove the theorem we only need to prove it for the case of the general linear group. Indeed, fix a faithful rational representation $\rho$ of the group $\bar{G}$. This representation identifies $\bar{G}$ with a subgroup of $\mathrm{GL}(V)$, where $V$ is the representation space of dimension $n=\operatorname{dim} \rho$. It is well known (see 34) that a base in $V$ can be chosen in such a way that after identification of $\operatorname{GL}(V)$ with $\mathrm{GL}(n, V)$ the split maximal torus $\bar{T}$ is contained in the diagonal subgroup $D(n, K)$, its normalizer $\bar{N}$ is contained in the monomial subgroup $N(n, K)$, and the unipotent radical of the standard Borel subgroup $U$ is contained in the upper unitriangular subgroup $U(n, K)$. Let $\widetilde{B}$ denote the standard Borel subgroup $B(n, K)=D(n, K) U(n, K)$ of $\mathrm{GL}(n, K)$. Then the image of any Borel double coset $\bar{B} w \bar{B}$ of $\bar{G}$ is contained in a Borel double coset $\widetilde{B} w \widetilde{B}$ of the general linear group $\operatorname{GL}(n, K)$. Vice versa, if the intersection of some class $\widetilde{B} w \widetilde{B}$ with $\bar{G}$ is nonempty (i.e., $w \in \bar{N}$ ), then this intersection coincides with the class $\bar{B} w \bar{B}$. Thus, the question 
about a typical Weyl factor $w$ for the group $\bar{G}$ is completely reduced to a similar question for the general linear group $\mathrm{GL}(n, K)$.

It remains to prove the theorem for the case of the general linear group. Consider the algorithm for finding $w$ in the Bruhat decomposition of an element of the general linear group, discussed in [34, §3]. Clearly, the condition that an element $y=\left(y_{i j}\right) \in \operatorname{GL}(n, K)$ belongs to a given Borel double coset $\widetilde{B} w \widetilde{B}$ is a set of algebraic conditions - equations and inequalities - in the entries of $y$. In fact, these conditions require that some minors of $y$ be equal to 0 , while some other minors be distinct from 0 . Let $f_{1}\left(y_{i j}\right), \ldots, f_{s}\left(y_{i j}\right)$ be the complete set of polynomials that determine the permutation $w$. As a matter of fact, this is the set of all proper minors of $y$ in such an order that the subsequent ones start to play a role if the values of some of the preceding ones are 0 .

For instance, first we look at whether $y_{n 1}$ is zero or not. If $y_{n 1}=0$, then we start to look at $y_{n 2}$ and $y_{n-1,1}$. If $y_{n 2}$ is also zero, then we start to look at $y_{n 3}$ and $y_{n-1,2}$. In turn, if $y_{n-1,1}$ is zero, then we start to look at $y_{n-1,2}$ and $y_{n-2,1}$, etc. On the other hand, if $y_{n 1} \neq 0$, then all of the above do not arise, but we start to look at the minor $\Delta_{n, n-1}^{12}(y)=y_{n 1} y_{n-1,2}-y_{n-1,1} y_{n 2}$. Now, if $\Delta_{n, n-1}^{12}(y)=0$, then we start to look at the next minors of the same size, namely at $\Delta_{n, n-1}^{13}(y)$ and $\Delta_{n, n-2}^{12}(y)$, etc. But if $\Delta_{n, n-1}^{12}(y) \neq 0$, then we start to look at the bottom left minor of the next size $\Delta_{n, n-1, n-2}^{123}$, etc., etc.

It only remains to apply the arguments from the previous paragraph to the matrices $y(\varepsilon)=x d(\varepsilon) x^{-1}$, where $\varepsilon \in K^{*}, x$ is a fixed matrix in $\operatorname{GL}(n, K)$, and $d(\varepsilon)=$ $\operatorname{diag}\left(\varepsilon^{m_{1}}, \ldots, \varepsilon^{m_{n}}\right)$ is a diagonal matrix. Every entry of the matrix $y(\varepsilon)$ is a Laurent polynomial in $\varepsilon$. Set $F_{1}(\varepsilon)=f_{1}\left(y_{i j}(\varepsilon)\right), \ldots, F_{s}(\varepsilon)=f_{s}\left(y_{i j}(\varepsilon)\right)$. Some of the Laurent polynomials $F_{1}, \ldots, F_{s}$, say the polynomials with indices $h_{1}, \ldots, h_{q}$, are zeros. All other polynomials have only finitely many zeros altogether. This means that for all $\varepsilon$ 's apart from a finite number of them, the matrices $y(\varepsilon)$ belong to the same Borel double coset, namely to the coset defined by the polynomial conditions $F_{h}=0$ for $h=h_{1}, \ldots, h_{q}$ and $F_{h} \neq 0$ otherwise.

Actually, as we shall see below, for a microweight $\omega$ all the nontrivial elements $x h_{\omega}(\varepsilon) x^{-1}$ belong to one and the same Bruhat double coset. In the Thesis of Semenov it was established that all the semisimple long root elements $x h_{\alpha}(\varepsilon) x_{-1}$ apart from at most three of them (the trivial one and at most two additional ones) belong to one and the same Bruhat double coset; see [31, 32, 22, 23, 71].

\section{§14. Bruhat DECOMPosition OF MICROWEIGHT ELEMENTS}

We are all set to finish the proof of Theorem 1 stated in the Introduction.

Indeed, let $x=u w v d$, where $u \in U, w \in W, v \in U_{w}^{-}$, and $d \in \bar{T}$, be the right reduced Bruhat decomposition of an element $x \in \bar{G}$. We start to calculate $y(\varepsilon)=x h_{\omega}(\varepsilon) x^{-1}$. First, look at $v h_{\omega}(\varepsilon) v^{-1}$. Write $v$ in the form $v=\prod x_{\alpha}\left(v_{\alpha}\right)$, where the product is taken over all $\alpha \in \Phi^{+}$, in the order described below. We recall that, since $v \in U_{w}^{-}$, a coefficient $v_{\alpha}$ may be distinct from 0 only for $\alpha \in \Phi^{+} \cap w^{-1} \Phi^{-}$. We arrange factors in the following order: first all factors corresponding to the roots in $\Sigma=\Sigma_{\omega}$, in any order - since $\Sigma$ is Abelian, these factors commute - then all factors corresponding to the roots in $\Delta=\Delta_{\omega}$, in any order. In the expression for $v h_{\omega}(\varepsilon) v^{-1}$ all factors belonging to $G(\Delta, K)$ disappear. Indeed, by the definition of a weight element, $h_{\omega}(\varepsilon)$ commutes with all $x_{\alpha}(\xi), \alpha \in \Delta$. On the other hand, $(\alpha, \omega)=1$ for all $\alpha \in \Sigma$, whence

$$
v h_{\omega}(\varepsilon) v^{-1}=\prod x_{\alpha}\left((1-\varepsilon) v_{\alpha}\right) h_{\omega}(\varepsilon), \quad \alpha \in \Sigma .
$$


Next, we look at the product $z(\varepsilon)=w v h_{\omega}(\varepsilon) v^{-1} w^{-1}$. It is well known; see 43, Theorem 2.5.8], that every element $w$ of the Weyl group $W(\Phi)$ may be uniquely expressed in the form $w=w^{\prime} w^{\prime \prime}$, where $l(w)=l\left(w^{\prime}\right)+l\left(w^{\prime \prime}\right), w^{\prime \prime} \in W(\Delta)$, and $w^{\prime}$ has the following property: $w^{\prime} \Delta^{+} \subseteq \Phi^{+}$. The element $w^{\prime \prime}$ permutes the roots in $\Sigma$. Thus, without loss of generality we may assume that $w^{\prime \prime}=e$ and $w=w^{\prime}$. It follows that

$$
z(\varepsilon)=\prod x_{w \alpha}\left( \pm(\varepsilon-1) v_{\alpha}\right) h_{w \omega}(\varepsilon), \quad \alpha \in \Sigma
$$

(the factors corresponding to the roots $\alpha \in \Sigma$ for which $w \alpha>0$ are trivial automatically). The set $w \Sigma$ is of the same structure as the set $\Sigma$ itself. In particular, it has a unique smallest root and all other roots in $w \Sigma$ are obtained from that root by consecutive additions of the roots $w \beta, \beta \in \Pi \cap \Delta$. In general, the roots $w \beta$ are not fundamental; nevertheless, by our condition on $w$, they are all positive.

This means that we can apply Proposition 3 to the vector consisting of the coordinates of $z(\varepsilon)$ and conclude that there exists a matrix $u^{\prime} \in U$ such that $\left(u^{\prime}\right)^{-1} z(\varepsilon) u^{\prime}$ equals

$$
\left(u^{\prime}\right)^{-1} z(\varepsilon) u^{\prime}=\prod x_{-\gamma}\left((\varepsilon-1) a_{\gamma}\right) h_{w \omega}(\varepsilon), \quad \gamma \in \Omega .
$$

Here the product is taken over a set $\Omega$ of pairwise strictly orthogonal positive roots contained in $-w \Sigma$ and the coefficients $a_{\gamma}$ do not depend on $\varepsilon$. The conjugating matrix $u^{\prime}$ does not depend on $\varepsilon$ and is a product of elementary factors $x_{\alpha}(*)$ taken over the roots of $\Phi^{+}$not orthogonal to at least one $\gamma \in \Omega$. Now, expressing $x_{-\gamma}\left((\varepsilon-1) a_{\gamma}\right)$ in terms of $w_{\gamma}(*)$ and $x_{\gamma}(*)$, and taking into account the fact that the unipotents corresponding to strictly orthogonal roots commute, we get

$z(\varepsilon)=u^{\prime} \prod x_{\gamma}\left((\varepsilon-1)^{-1} a_{\gamma}^{-1}\right) \prod w_{\gamma}\left(-(\varepsilon-1)^{-1} a_{\gamma}^{-1}\right) \prod x_{\gamma}\left((\varepsilon-1)^{-1} a_{\gamma}^{-1}\right) h_{w \omega}(\varepsilon)\left(u^{\prime}\right)^{-1}$,

where all products are taken over $\gamma \in \Omega$. Conjugating this last expression by $u$ we see that $y(\varepsilon) \in \bar{B}\left(\prod w_{\gamma}\right) \bar{B}$, as claimed. This finishes the proof of the theorem.

\section{$\S 15$. Concluding Remarks}

In the present paper we classified the Weyl factors appearing in the Bruhat decomposition of microweight elements. This result was originally motivated by applications to overgroups of maximal split tori in extended Chevalley groups; see [16, 17, 78, Presently, the author and Vladimir Nesterov have returned to the study of microweight elements in connection with the following problem, mentioned in [19].

Problem 1. Describe the orbits of a Chevalley group $G(\Phi, K)$ acting by simultaneous conjugations on pairs of microweight tori

$$
X, Y \sim\left\{h_{\omega}(\varepsilon) \mid \varepsilon \in K^{*}\right\}, \quad \omega \in P\left(\Phi^{\vee}\right),
$$

and the corresponding spans.

For the majority of the real world applications, the following qualitative result in this direction would already suffice. After that one could apply the known results by Timmesfeld, Bashkirov, and others, classifying the subgroups that contain quadratic elements of small residue.

Problem 2. Establish that a subgroup generated by a pair of noncommuting microweight tori $X, Y \sim\left\{h_{\omega}(\varepsilon) \mid \varepsilon \in K^{*}\right\}$ contains a small unipotent element.

To simplify calculations in these problems, one should first solve the following problem, mentioned in the Introduction.

Problem 3. Determine the shape of Borel factors in the Bruhat decomposition of the elements of a microweight torus $X \sim\left\{h_{\omega}(\varepsilon) \mid \varepsilon \in K^{*}\right\}$. 
Below we allude to yet another variation of this theme, which can facilitate calculations arising in the solution of Problems 1 and 2. Namely, we propose to determine the small Bruhat cells [21, 29, 55] containing microweight elements, rather than the ordinary cells.

Problem 4. Find the small Bruhat cells hosting the elements of a microweight torus $X \sim\left\{h_{\omega}(\varepsilon) \mid \varepsilon \in K^{*}\right\}$.

I greatly appreciate the help of Chris Parker and Elizaveta Dybkova, who carefully read the first [English/Russian] versions of the manuscript and suggested a huge number of improvements.

\section{REFERENCES}

[1] Z. I. Borevich, Description of the subgroups of the general linear group that contain the group of diagonal matrices, Zap. Nauchn. Sem. Leningrad. Otdel. Mat. Inst. Steklov. (LOMI) 64 (1976), 12-29; English transl., J. Soviet Math. 17 (1981), no. 2, 1718-1730. MR0447422 (56:5734)

[2] Z. I. Borevich and N. A. Vavilov, Subgroups of the general linear group over a semilocal ring that contain a group of diagonal matrices, Trudy Mat. Inst. Steklov. 148 (1978), 43-57; English transl. in Proc. Steklov Inst. Math. 1980, no. 4. MR0558939 (81g:20085)

[3] A. Borel, Properties and linear representations of Chevalley groups, Seminar on Algebraic Groups and Related Finite Groups (Inst. Adv. Study, Princeton, NJ, 1968/69), Lecture Notes in Math., vol. 131, Springer-Verlag, Berlin-New York, 1970, pp. 1-55. MR0258838 (41:3484)

[4] N. Bourbaki, Lie groups and Lie algebras. Chapters 4-6, Springer-Verlag, Berlin, 2002. MR1890629 (2003a:17001)

[5] , Lie groups and Lie algebras. Chapters 7-9, Springer-Verlag, Berlin, 2005. MR.2109105 (2005h:17001)

[6] N. A. Vavilov, On subgroups of the general linear group over a semilocal ring containing a group of diagonal matrices, Vestnik Leningrad. Univ. Mat. Mekh. Astronom. 1981, vyp. 1, 10-15; English transl. in Vestnik Leningrad Univ. Math. 14 (1982), no. 1. MR0613803 (82g:20073)

[7] _ Bruhat decomposition for subgroups containing the group of diagonal matrices. I, II, Zap. Nauchn. Sem. Leningrad. Otdel. Mat. Inst. Steklov. (LOMI) 103 (1980), 20-30; ibid. 114 (1982), 50-61; English transl. in J. Soviet Math. 24 (1984), no. 4; ibid 27 (1984), no. 4. MR0618491 (82g:20072) MR0669559(84e:20050)

[8] Subgroups of the special linear group which contain the group of diagonal matrices. I-V, Vestnik Leningrad. Univ. Mat. Mekh. Astronom. 1985, vyp. 4, 3-7; 1986, vyp. 1, 10-15; 1987, vyp. $2,3-8$; 1988, vyp. 3, 10-15; 1993, vyp. 2, 10-15; English transl. in Vestnik Leningrad Univ. Math. 18 (1985), no. 4; 19 (1986), no. 1; 20 (1987), no. 2; 21 (1988), no. 3, 7-15; 26 (1993), no. 2, 6-9. MR0827955 (87e:20081) MR.0841489 (87j:20074) MR0926257 (89b:20097) $\quad$ MR0974786 (90a:20088) MR1370226 (96k:20089)

[9] , Bruhat decomposition of one-dimensional transformations, Vestnik Leningrad. Univ. Mat. Mekh. Astronom. 1986, vyp. 3, 14-20; English transl. in Vestnik Leningrad Univ. Math. 19 (1986), no. 3. MR0867389 (88g:20094)

[10] _ Weight elements of Chevalley groups, Dokl. Akad. Nauk SSSR 298 (1988), no. 3, 524-527; English transl., Soviet Math. Dokl. 37 (1988), no.1, 92-95. MR0925952 (88m:20093)

[11] Conjugacy theorems for subgroups of extended Chevalley groups that contain split maximal tori, Dokl. Akad. Nauk SSSR 299 (1988), no. 2, 269-272; English transl., Soviet Math. Dokl. 37 (1988), no. 2, 360-363. MR0943230 (89e:20081)

[12] _ Bruhat decomposition for long root semisimple elements in Chevalley groups, Rings and Modules. Limit Theorems of Probability Theory, No. 2, Leningrad. Univ., Leningrad, 1988, pp. 1839. (Russian) MR0974130(89k:20057)

[13] _ Bruhat decomposition of two-dimensional transformations, Vestnik Leningrad. Univ. Mat. Mekh. Astronom. 1989, vyp. 3, 3-7; English transl., Vestnik Leningrad Univ. Math. 22 (1989), no. 3, 1-6. MR 1055331 (91c:20057)

[14] _ Root semisimple elements and triples of root unipotent subgroups in Chevalley groups, Problems in Algebra, No. 4, "Universitetskoe", Minsk, 1989, pp. 162-173. (Russian) MR1011925 (90i:20046)

[15] , Subgroups of split classical groups, Trudy Mat. Inst. Steklov. 183 (1990), 29-42; English transl., Proc. Steklov Inst. Math. 1991, no. 4, 27-41. MR.1092012 (92d:20065)

[16] Subgroups of Chevalley groups containing a maximal torus, Trudy Leningrad. Mat. Obshch. 1 (1990), 64-109; English transl., Amer. Math. Soc. Transl. Ser. 2, vol. 155, Amer. Math. Soc., Providence, RI, 1993, pp. 59-100. MR/1104207 (92f:20046) 
[17] Unipotent elements in subgroups of extended Chevalley groups that contain a split maximal torus, Dokl. Akad. Nauk 328 (1993), no. 5, 536-539; English transl., Russian Acad. Sci. Dokl. Math. 47 (1993), no. 1, 112-116. MR1218959 (94k:20083)

[18] _ Subgroups of group $\mathrm{SL}_{n}$ over semilocal ring, Zap. Nauchn. Sem. S.-Peterburg. Otdel. Mat. Inst. Steklov. (POMI) (to appear). (Russian)

[19] _ Geometry of 1-tori in $\mathrm{GL}_{n}$, Algebra i Analiz 19 (2007), no. 3, 119-150; English transl., St. Petersburg Math. J. 19 (2008), no. 3, 407-429. MR2340708 (2008g:20115)

[20] N. A. Vavilov and E. V. Dybkova, Subgroups of the general symplectic group containing the group of diagonal matrices. I, II, Zap. Nauchn. Sem. Leningrad. Otdel. Mat. Inst. Steklov. (LOMI) 103 (1980), 31-47; 132 (1983), 44-56; English transl. in J. Soviet Math. 24 (1984), no. 4; 30 (1985), no. 1. MR0618492 (82h:20054), MR0717571 (85i:20049)

[21] N. A. Vavilov and M. Yu. Mitrofanov, Intersection of two Bruhat cells, Dokl. Akad. Nauk 377 (2001), no. 1, 7-10; English transl., Dokl. Math. 63 (2001), no. 2, 149-151. MR1833978 (2003b:20065)

[22] N. A. Vavilov and A. A. Semenov, Bruhat decomposition for long root tori in Chevalley groups, Zap. Nauchn. Sem. Leningrad. Otdel. Mat. Inst. Steklov. (LOMI) 175 (1989), 12-23; English transl., J. Soviet Math. 57 (1991), no. 6, 3453-3458. MR.1047233 (91b:20060)

[23] _ Long root semisimple elements in Chevalley groups, Dokl. Akad. Nauk 338 (1994), no. 6, 725-727; English transl., Russian Acad. Sci. Dokl. Math. 50 (1995), no. 2, 325-329. MR1311310 (96c:20084)

[24] M. Goto and F. D. Grosshans, Semisimple Lie algebras, Lecture Notes in Pure Appl. Math., vol. 38, Marcel Dekker, Inc., New York-Basel, 1978. MR0573070 (58:28084)

[25] E. B. Dynkin, Semisimple subalgebras of semisimple Lie algebras, Mat. Sb. (N.S.) 30 (1952), 349462. (Russian) MR0047629 (13:904c)

[26] A. E. Zalesskiı̌, Linear groups, Itogi Nauki i Tekhniki. Algebra. Topology. Geometry, vol. 21, VINITI, Moscow, 1983, pp. 135-182; English transl., J. Soviet Math. 31 (1985), no. 3, 2974-3004. MR0724616 (86b:20051)

[27] V. V. Kashin, Orbits of an adjoint and co-adjoint action of Borel subgroups of a semisimple algebraic group, Problems in Group Theory and Homological Algebra, Yaroslav. Gos. Univ., Yaroslavl', 1990, pp. 141-158. (Russian) MR1169975 (93m:20058)

[28] A. S. Kondrat'ev, Subgroups of finite Chevalley groups, Uspekhi Mat. Nauk 41 (1986), no. 1, 57-96; English transl., Russian Math. Surveys 41 (1986), no. 1, 65-118. MR0832410 (87g:20078)

[29] M. Yu. Mitrofanov, The role of matroids in the description of thin Bruhat cells, Zap. Nauchn. Sem. S.-Peterburg. Otdel. Mat. Inst. Steklov. (POMI) 319 (2004), 244-260; English transl., J. Math. Sci. (N. Y.) 134 (2006), no. 6, 2572-2579. MR2117859 (2005j:05019)

[30] V. L. Popov, Closed orbits of Borel subgroups, Mat. Sb. (N. S.) 135 (1988), no. 3, 385-402; English transl., Math. USSR-Sb. 63 (1989), no. 2, 375-392. MR0937648 (89c:14073)

[31] A. A. Semenov, Bruhat decomposition of root semisimple subgroups in the special linear group, Zap. Nauchn. Sem. Leningrad. Otdel. Mat. Inst. Steklov. (LOMI) 160 (1987), 239-246; English transl., J. Soviet Math. 52 (1990), no. 3, 3178-3185. MR0906861 (88g:20093)

[32] _ Bruhat decomposition for long root semisimple tori in Chevalley groups, Candidate Diss., S.-Peterburg. Univ., St. Petersburg, 1991.

[33] T. A. Springer and R. Steinberg, Conjugacy classes, Seminar on Algebraic Groups and Related Finite Groups (Princeton, NJ, 1968/69), Lecture Notes in Math., vol. 131, Springer-Verlag, Berlin, 1970, pp. 167-266. MR0268192 (42:3091)

[34] R. Steinberg, Lectures on Chevalley groups, Yale Univ., New Haven, Conn., 1968. MR0466335 $(68: 6215)$

[35] J. E. Humphreys, Introduction to Lie algebras and representation theory, Grad. Texts in Math., No. 9, Springer-Verlag, New York-Berlin, 1978. MR0499562 (81b:17007)

[36] C. Chevalley, Sur certains groupes simples, Tôhoku Math. J. (2) 7 (1955), 14-66. MR0073602 $(17: 457 \mathrm{c})$

[37] E. Abe, Chevalley groups over local rings, Tôhoku Math. J. (2) 21 (1969), no. 3, 474-494. MR0258837 (41:3483)

[38] M. Aschbacher, Some multilinear forms with large isometry groups, Geom. Dedicata 25 (1988), no. 1-3, 417-465. MR0925846 (89c:20067)

[39] H. Azad, M. Barry, and G. M. Seitz, On the structure of parabolic subgroups, Comm. Algebra 18 (1990), 551-562. MR.1047327(91d:20048)

[40] S. Berman and R. Moody, Extensions of Chevalley groups, Israel J. Math. 22 (1975), no. 1, 42-51. MR0390077(52:10903) 
[41] M. Brion, Représentations exceptionnelles des groupes semi-simples, Ann. Sci. École Norm. Sup. (4) 18 (1985), 345-387. MR0816368 (87e:14043)

[42] H. Bürgstein and W. H. Hesselink, Algorithmic orbit classification for some Borel group actions, Compositio Math. 61 (1987), no. 1, 3-41. MR0879187 (88k:20069)

[43] R. W. Carter, Simple groups of Lie type, John Wiley and Sons, Inc., New York, 1989. MR1013112 (90g:20001)

[44] , Conjugacy classes in the Weyl group, Compositio Math. 25 (1972), no. 1, 1-59. MR0318337 $(47: 6884)$

[45] _ Finite groups of Lie type: Conjugacy classes and complex characters, Wiley and Sons, Inc., New York, 1985. MR0794307 (87d:20060)

[46] A. M. Cohen and B. N. Cooperstein, The 2-spaces of the standard $\mathrm{E}_{6}(q)$-module, Geom. Dedicata 25 (1988), no. 1-3, 467-480. MR0925847 (89c:51013)

[47] D. I. Deriziotis, Conjugacy classes and centralizers of semisimple elements in finite groups of Lie type, Vorlesungen Fachbereich Math. Univ. Essen, no. 11, Univ. Essen, Essen, 1984. MR0742140 (85k:20139)

[48] E. Ellers and N. Gordeev, Intersection of conjugacy classes with Bruhat cells in Chevalley groups, Pacific J. Math. 214 (2004), 245-261. MR2042932 (2004m:20091)

[49] P. Gilkey and G. M. Seitz, Some representations of exceptional Lie algebras, Geom. Dedicata 25 (1988), no. 1-3, 407-416. MR0925845 (89h:20056)

[50] A. L. Harebov and N. A. Vavilov, On the lattice of subgroups of Chevalley groups containing a split maximal torus, Comm. Algebra 24 (1996), no. 1, 109-133. MR1370526 (97a:20077)

[51] S. Haris, Some irreducible representations of exceptional algebraic groups, Amer. J. Math. 93 (1971), no. 1, 75-106. MR0279103 (43:4829)

[52] W. Hesselink, A classification of the nilpotent triangular matrices, Compositio Math. 55 (1985), no. 1, 89-133. MR0791648 (87a:20049)

[53] J. G. M. Mars, Les nombres de Tamagawa de certains groupes exceptionnels, Bull. Soc. Math. France 94 (1966), 97-140. MR0213363 (35:4227)

[54] H. Matsumoto, Sur les sous-groupes arithmétiques des groupes semi-simples déployés, Ann. Sci. École Norm. Sup. (4) 2 (1969), 1-62. MR0240214 (39:1566)

[55] M. Mitrofanov and N. Vavilov, Overgroups of the diagonal subgroup via small Bruhat cells, Algebra Colloq. (to appear).

[56] Ch. Parker and G. E. Röhrle, Minuscule representations, Preprint SFB 343, no. 72, Univ. Bielefeld, 1993.

[57] _ The restriction of minuscule representations to parabolic subgroups, Math. Proc. Cambridge Philos. Soc. 135 (2003), 59-79. MR1990832 (2005c:20073)

[58] E. B. Plotkin, A. A. Semenov, and N. A. Vavilov, Visual basic representations: an atlas, Internat. J. Algebra Comput. 8 (1998), 61-97. MR:1492062 (98m:17010)

[59] V. L. Popov, A finiteness theorem for parabolic subgroups of fixed modality, Indag. Math. (N.S.) 8 (1997), no. 1, 125-132. MR.1617826 (99k:20095)

[60] V. L. Popov and G. Röhrle, On the number of orbits of a parabolic subgroup on its unipotent radical, Algebraic Groups and Lie Groups, Austral. Math. Soc. Lect. Ser., vol. 9, Cambridge Univ. Press, Cambridge, 1997, pp. 297-320. MR.1635688 (99f:14063)

[61] R. Richardson, Finiteness theorems for orbits of algebraic groups, Indag. Math. 47 (1985), 337-344. MR0814886 (87e:14044)

[62] R. Richardson, G. E. Röhrle, and R. Steinberg, Parabolic subgroups with abelian unipotent radical, Invent. Math. 110 (1992), no. 3, 649-671. MR1189494 (93j:20092)

[63] G. E. Röhrle, Orbits in internal Chevalley modules, Groups, Combinatorics and Geometry (Durham, 1990), London Math. Soc. Lecture Note Ser., vol. 165, Cambridge Univ. Press, Cambridge, 1992, pp. 311-315. MR1200268

[64] 80-115. MR 1219660 (94d:20053)

[65] _ On extraspecial parabolic subgroups, Linear Algebraic Groups and their Representations (Los Angeles, CA, 1992), Contemp. Math., vol. 153, Amer. Math. Soc., Providence, RI, 1993, pp. 143-155. MR1247502 (94k:20082)

[66] _ Parabolic subgroups of positive modality, Geom. Dedicata 60 (1996), 163-186. MR1384426 (97c:20070)

[67] , A note on the modality of parabolic subgroups, Indag. Math. 8 (1997), no. 4, 549-559. MR.1621854 (99e:20060)

[68] - On the modality of parabolic subgroups of linear algebraic groups, Manuscripta Math. 98 (1999), 9-20. MR1669615 (2000f:20077) 
[69] G. M. Seitz, Subgroups of finite groups of Lie type, J. Algebra 61 (1979), no. 1, 16-27. MR0554848 (81d:20039)

[70] G. B. Seligman, Modular Lie algebras, Ergeb. Math. Grenzgeb., Bd. 40, Springer-Verlag New York, Inc., New York, 1967. MR0245627 (39:6933)

[71] A. A. Semenov and N. A. Vavilov, Halbeinfache Wurzelelemente in Chevalley-Gruppen, Preprint no. 2, Univ. Bielefeld, 1994, 11 pp.

[72] T. A. Springer, Linear algebraic groups, Progr. Math., vol. 9, Birkhäuser, Boston, MA, 1981. MR0632835 (84i:20002)

[73] M. R. Stein, Stability theorems for $\mathrm{K}_{1}, \mathrm{~K}_{2}$ and related functors modeled on Chevalley groups, Japan. J. Math. (N.S.) 4 (1978), no. 1, 77-108. MR0528869 (81c:20031)

[74] J. Tits, Groupes semi-simples isotropes, Colloq. Théorie des Groupes Algébriques (Bruxelles, 1962), Gauthier-Villars, Paris, 1962, pp. 137-147. MR0148667 (26:6174)

[75] _ Normalisateurs de tores. I. Groupes de Coxeter étendus, J. Algebra 4 (1966), no. 1, 96-116. MR0206117 (34:5942)

[76] N. A. Vavilov, Structure of Chevalley groups over commutative rings, Nonassociative Algebras and Related Topics (Hiroshima, 1990), World Sci. Publ., River Edge, NJ, 1991, pp. 219-335. MR1150262 (92k:20090)

[77] Weight elements of Chevalley groups, Preprint no. 35, Univ. Warwick, 1994, 46 pp.

[78] _ Intermediate subgroups in Chevalley groups, Groups of Lie Type and their Geometries (Como, 1993), London Math. Soc. Lecture Note Ser., vol. 207, Cambridge Univ. Press, Cambridge, 1995, pp. 233-280. MR1320525 (96c:20085)

[79] _ Unipotent elements in subgroups which contain a split maximal torus, J. Algebra 176 (1995), 356-367. MR1351614(96i:20060)

[80] _ A third look at weight diagrams, Rend. Sem. Mat. Univ. Padova 104 (2000), 201-250. MR:1809357 (2001i:20099)

[81] _ Do it yourself structure constants for Lie algebras of types $E_{l}$, Zap. Nauchn. Sem. S.Peterburg. Otdel. Mat. Inst. Steklov. (POMI) 281 (2001), 60-104; English transl., J. Math. Sci. (N. Y.) 120 (2004), no. 4, 1513-1548. MR1875718(2002k:17022)

[82] N. A. Vavilov and E. B. Plotkin, Chevalley groups over commutative rings. I. Elementary calculations, Acta Appl. Math. 45 (1996), 73-113. MR1409655 (97h:20056)

Department of Mathematics and Mechanics, St. Petersburg State University, Universitetskil Prospekt 28, Staryŭ Peterhof, St. Petersburg 198504, Russia

E-mail address: nikolai-vavilov@yandex.ru

Received 8/NOV/2006

Translated by THE AUTHOR 